\title{
Active Feedback Technique for RF Channel Selection in Front-End Receivers
}

\author{
Shadi Youssef, Student Member, IEEE, Ronan van der Zee, Member, IEEE, \\ and Bram Nauta, Member, IEEE
}

\begin{abstract}
Co-existence problems in a mobile terminal environment pose strict requirements on the linearity of a front-end receiver. In this paper, active feedback is explored as a means to relax such requirements by providing channel selectivity as early as possible in the receiver chain. The proposed receiver architecture addresses some of the most common problems of integrated RF filters, while maintaining their inherent tunability. Through a simplified and intuitive analysis, the operation of the receiver is examined and the design parameters affecting the filter characteristics, such as bandwidth and stop-band rejection, are determined. A systematic procedure for analyzing the linearity of the receiver reveals the possibility of LNA distortion canceling, which decouples the trade-off between noise, linearity and harmonic radiation. A prototype designed in a standard $65 \mathrm{~nm}$ CMOS process occupies $<0.06 \mathrm{~mm}^{2}$ and utilizes an RF channel-select filter with a 1-to- $2.5 \mathrm{GHz}$ tunable center frequency to achieve $48 \mathrm{~dB}$ of stop-band rejection and a wideband IIP3 $>+12 \mathrm{dBm}$.
\end{abstract}

\section{Index Terms}

Integrated RF filtering, RF channel selection, interference rejection, active feedback receiver, frequency translation loop, down-conversion, up-conversion, passive mixers, distortion canceling, CMOS. 


\section{INTRODUCTION}

With the explosive growth in available wireless standards, next generation wireless transceivers are required to have multi-mode capabilities in order to meet cost, size and time-to-market demands. Furthermore, newly emerging concepts like cognitive radio aim for better utilization of the available radio spectrum through smart sensing and sharing of frequency channels between multiple users [1]. In both cases, a wireless transceiver requires the flexibility of operating within a wide range of frequencies, while simultaneously being able to deal with co-existence problems where, for instance, a receiver tries to detect a weak signal in the vicinity of at least one active transmitter. As a result, high linearity requirements, with IIP3 values as high as 30 -to-40dBm [2], are needed to prevent desensitization of the receiver.

In theory, such high linearity requirements can be met through RF filtering to only receive the desired frequency channel while rejecting all interferers that may be present at the antenna. Traditionally, high-Q filtering has been implemented by using off-chip components such as SAW filters [3]. In addition to being bulky and expensive, SAW filters are only suitable for selecting a fixed range of frequencies due to their lack of tunability. Therefore, they can only be used for selecting a complete application band at RF and/or IF channel selection where the desired channel is always down-converted to the same frequency.

In recent years, there has been renewed interest in N-path filtering techniques [4] due to their suitability for integration in CMOS. In their most recent forms [5]-[8], these filters utilize the impedance transformation property of passive mixers, where a baseband impedance with low-pass (or high-pass) characteristics is up-converted to RF to achieve a high-Q band-pass (or notch) filter with a programmable center frequency through a clock. As such, this technique is suitable for addressing both linearity and flexibility requirements in wide-band and cognitive radio applications. Although quite attractive, these passive mixer filters suffer from several drawbacks that limit their usefulness and/or performance in practice. These include the need for large capacitance/die area, limited stop-band rejection, and a trade-off between noise, linearity and harmonic radiation. In [9], we presented an alternative approach for providing $R F$ channel selectivity via active feedback. The approach overcomes the aforementioned drawbacks of passive mixer filters while retaining their inherent flexibility. In this paper, we extend our work by providing system analysis to determine the main design parameters affecting the filter 
characteristics, and present a systematic procedure for examining the overall linearity of the system. Furthermore, additional simulation and measurement results are presented.

Section II discusses the problems of integrated RF filters and section III introduces the active feedback receiver architecture to address these issues. In section IV, the receiver is analyzed and the filter transfer function is derived. Section $\mathrm{V}$ discusses the noise and linearity performance of the proposed architecture, and a robust mechanism for distortion canceling is introduced. Section VI presents the designed chip prototype, section VII discusses simulation and measurement results, and section VIII concludes this paper.

\section{OPERATION AND LIMITATIONS OF INTEGRATED \\ RF FILTERING}

A simplified block diagram of a passive mixer band-pass filter is shown in Fig. 1. Since there is no isolation between the two sides of the mixer, the low-pass filter (LPF) connected to the mixer is transformed at the RF input into a high-Q band-pass filter centered around the local oscillator $(\mathrm{LO})$ frequency driving the mixer $\left(\omega_{\mathrm{LO}}\right)[10]$. The square wave LO signal needed for driving the switch also means that scaled versions of the band-pass filter appear around the harmonics of $\mathrm{LO}\left(2 \omega_{\mathrm{LO}}, 3 \omega_{\mathrm{LO}}, .\right.$. etc. $)$. Because these higher harmonic replicas are usually undesirable, one can adopt a double-balanced design to eliminate even order replicas, as well as harmonic rejection mixers to eliminate the 3-rd and 5-th order replicas [11], [12].

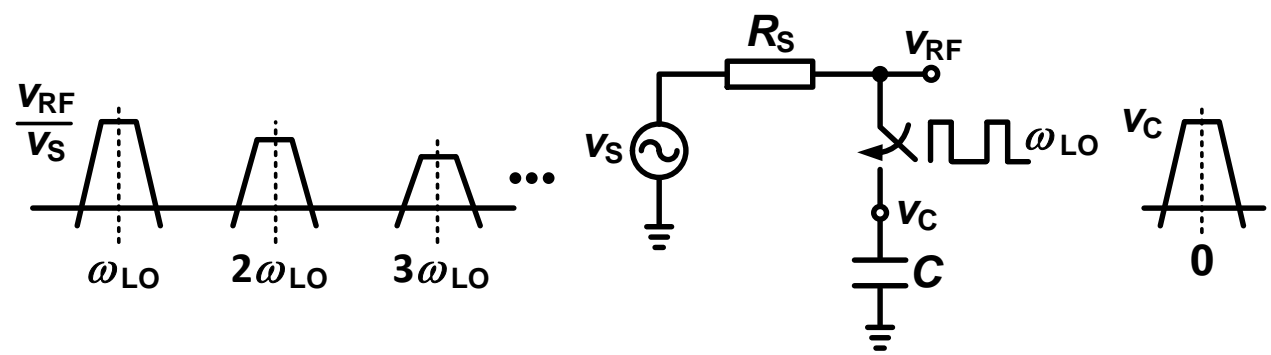

Fig. 1: Concept of integrated RF filtering via the impedance transformation property of a passive mixer.

One problem of these structures relates to the typical requirements of a front-end receiver in terms of input matching and noise. Generally speaking, both requirements lead to low resistance levels at the RF side of a receiver chain. Therefore, the RC product required for RF channel 
filtering results in large capacitors, and, consequently, a large die area that does not scale very well with technology. Typical capacitance values required in integrated filter designs are in the range of hundreds of picofarads [7], [8].

In addition, the maximum achievable filter rejection is limited by the on-resistance of the switches of the passive mixer. As shown in Fig. 2, for frequency offsets much larger than the LO frequency, the capacitor acts as a short circuit and maximum stop-band rejection at the RF side is limited by the voltage divider formed between the source resistance and the switch resistance. This problem is further exacerbated by the low value of source resistance available at RF as previously explained. To mitigate this issue, one can step-up the source resistance using an off-chip RF transformer [7]. However, the use of such bulky components contradicts the aim of achieving integrated high-Q RF filtering. Even with the use of an RF transformer, large switches are typically needed to achieve moderate rejection values $(5 \Omega$ switches for $16 \mathrm{~dB}$ rejection [7]). This directly translates to more parasitic capacitance in the switches and higher power consumption in the LO buffers.
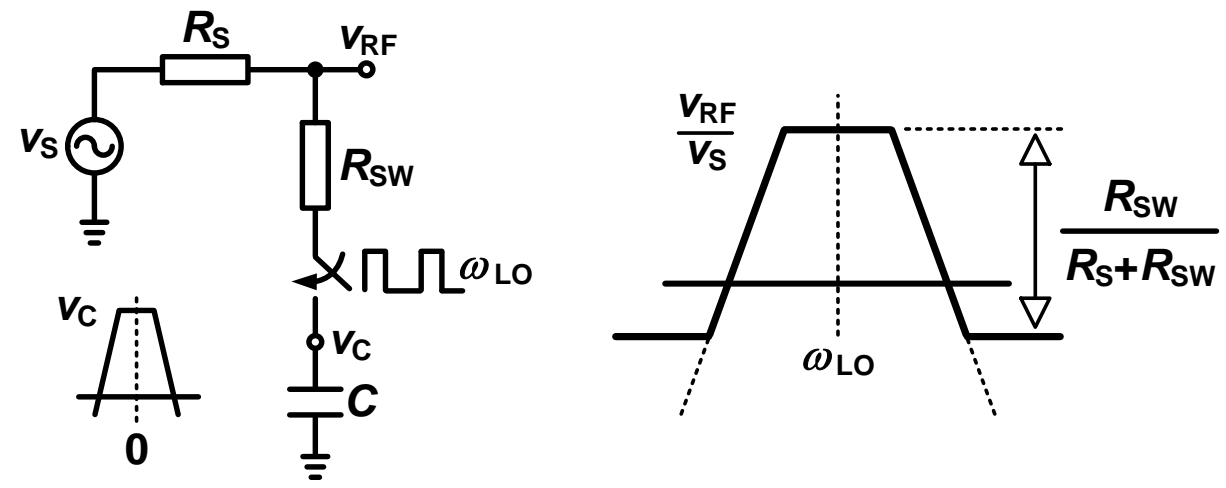

Fig. 2: Limitation on maximum stop-band rejection that can be achieved in integrated RF filters.

Furthermore, the position of the filter along the RF part of the receiver chain entails a basic trade-off. Filtering prior to the LNA [6] or eliminating it altogether [13] improves linearity at the expense of noise and switching harmonics being injected directly at the antenna node. Conversely, an LNA first architecture offers an opposite trade-off.

In this work, RF channel selection based on an active feedback frequency translation loop is presented. The proposed receiver architecture aims to provide channel selectivity as early as possible in the receiver chain to reduce distortion due to interferers, or equivalently, relax (or 
possibly eliminate) the receiver filtering requirements for a given application. As such, the work presented in this paper targets one of the key co-existence problems in radio receiver design. The receiver is shown to result in a highly compact and tunable design that mitigates the performance limitations of integrated RF filters discussed above [9], namely: large capacitance/die area, limited stop-band rejection and the trade-off between noise, linearity and harmonic radiation. Other issues that are equally important in the context of co-existence include spurious responses due to harmonic mixing, phase noise and even-order distortion. However, since the proposed architecture poses no specific requirements in relation to these issues, they are viewed as orthogonal problems. That is, techniques available for addressing harmonic mixing [11], [12], phase noise [11], [12], or even-order distortion [14], [15] can be employed equally well in an active feedback receiver, and are therefore outside the scope of this paper.

\section{Active Feedback Filtering Concept}

Figure 3 shows a block diagram of the proposed architecture. All signals present at the antenna (desired plus interferers) are amplified using a low noise amplifier (LNA), then down-converted for further amplification. Along the feedback path, the desired signal bandwidth, now centered at DC, is rejected using a high pass filter (HPF), while all interferers are up-converted once again and subtracted at the LNA output. As a result, the transfer function from the LNA input to the IF output provides gain for the desired signal and suppression for interferers, effectively creating an RF channel-select filter centered around LO.

Since filtering is chosen to be performed after the LNA, filter noise and harmonic radiation are not a major concern as previously explained. However, the LNA now needs to handle interferers prior to suppression, thus determining the overall linearity of the receiver chain. A robust way to cancel LNA distortion is examined in section V.

To demonstrate the properties and benefits of such an architecture, the gain and filter transfer function are first derived in the following section.

\section{Analysis of Active Feedback Receiver}

\section{A. RF-to-IF Gain}

A more detailed block diagram that captures the essential characteristics of the proposed architecture is shown in Fig. 4. In the forward path, the LNA is a transconductor $G_{\ln a}$ that 


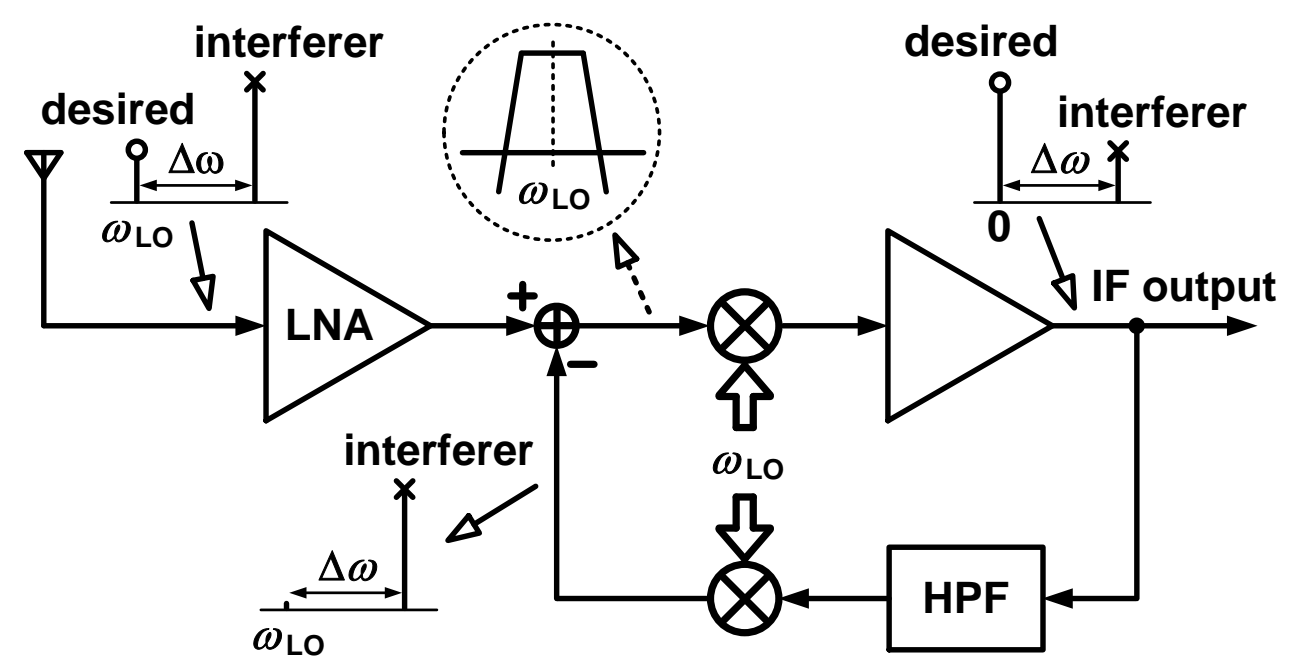

Fig. 3: Active feedback receiver architecture.

drives a passive mixer followed by a transimpedance amplifier to improve in-band linearity [12]. The feedback loop is implemented in a shunt-shunt fashion, where the IF output voltage is sensed, filtered and an RF current is fed back through a passive mixer driven by the feedback transconductor $G_{\mathrm{fb}}$. The HPF is a first order filter with a corner frequency $\omega_{\text {hpf }}$. Whether the loop rejects the desired signal prior to or after V-to-I conversion in $G_{\mathrm{fb}}$ does not change the resulting filter transfer function, but has a crucial effect on noise and distortion as will be shown in section V. Including the output impedance of both transconductors would significantly complicate the analysis, but since both down- and up-conversion operations are performed via current commutating mixers, the driving impedance at one side of the mixer is typically much higher than the load impedance at the other side of the mixer. Consequently, neglecting one or both driving impedances has a negligible effect on the operation of the circuit. The choice to only include the LNA output impedance $Z_{o}(\omega)$ will be motivated in section IV-B.

The RF-to-IF gain of the receiver can be written as

$$
A_{\mathrm{RF}-\mathrm{IF}}(\omega)=\frac{v_{o}(\Delta \omega)}{v_{\text {in }}(\omega)}=-G_{\mathrm{lna}} Z_{\mathrm{CL}}(\omega) A_{\mathrm{mix}} A_{v}(\Delta \omega)
$$

where $\Delta \omega$ is the frequency offset from LO, $A_{\text {mix }}$ is the current conversion gain of the mixer and is assumed to be equal for both up- and down-conversion mixers, and $Z_{\mathrm{CL}}(\omega)$ can be defined as an effective RF impedance seen at the output of the LNA in closed loop operation, and is given 


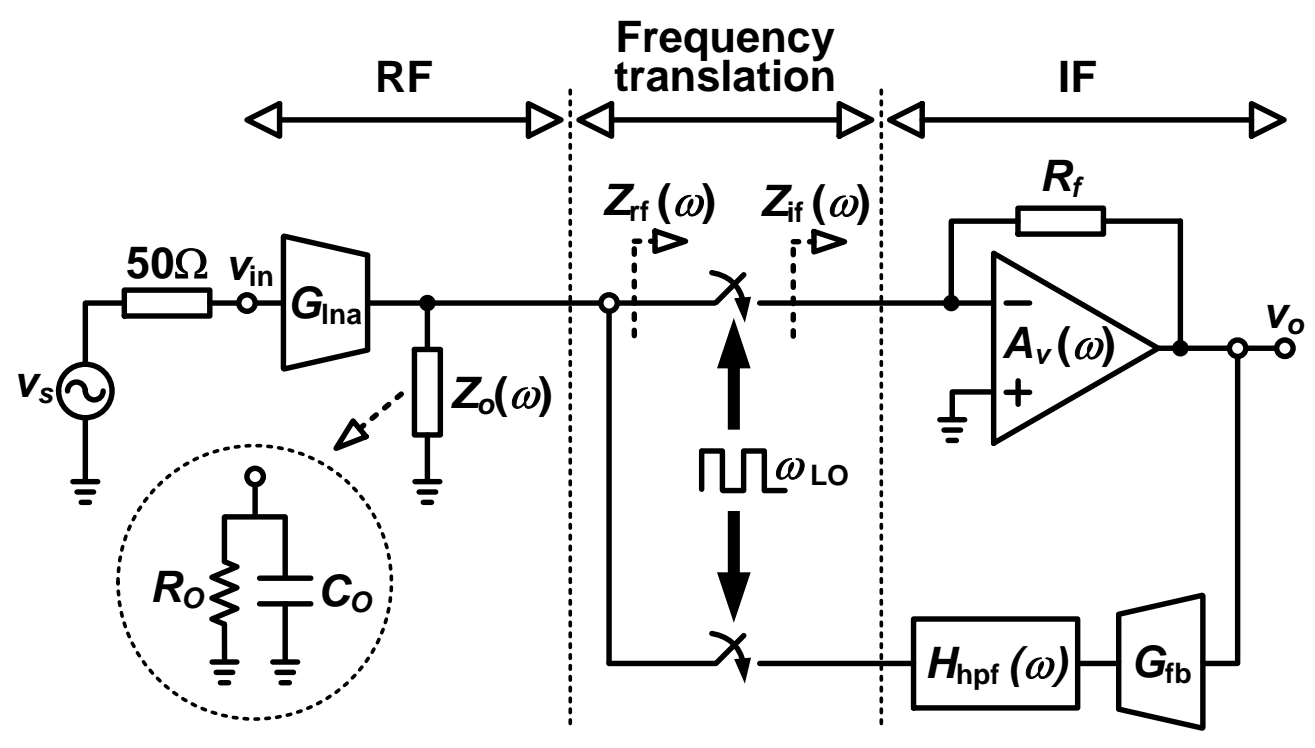

Fig. 4: A detailed block diagram of the active feedback receiver for transfer function derivation.

by

$$
Z_{\mathrm{CL}}(\omega)=Z_{\mathrm{OL}}(\omega) \frac{1}{1+T(\omega)}=\frac{Z_{o}(\omega) Z_{\mathrm{if}}(\Delta \omega)}{Z_{o}(\omega)+Z_{\mathrm{rf}}(\omega)} \frac{1}{1+T(\omega)}
$$

where $Z_{\mathrm{rf}}(\omega)$ is the RF impedance seen through the down-conversion mixer [10]

$$
Z_{\mathrm{rf}}(\omega)=R_{\mathrm{SW}}+A_{\mathrm{mix}}^{2} Z_{\mathrm{if}}(\Delta \omega)=R_{\mathrm{Sw}}+A_{\text {mix }}^{2} \frac{R_{f}}{1+A_{v}(\Delta \omega)}
$$

and $T(\omega)$ is the active feedback loop gain and is equal to

$$
T(\omega)=G_{\mathrm{fb}} \frac{Z_{o}(\omega) Z_{\mathrm{if}}(\Delta \omega)}{Z_{o}(\omega)+Z_{\mathrm{rf}}(\omega)} A_{\mathrm{mix}}^{2} A_{v}(\Delta \omega) H_{\mathrm{hpf}}(\Delta \omega)
$$

Note that the expressions in (3) and (4) are obtained by considering only the down-/up-converted gain due to the fundamental component of the $\mathrm{LO}$, and assuming that the version of the signal upconverted by the down-conversion mixer is filtered out by the loop before being down-converted by the up-conversion mixer.

For the desired signal, the following assumptions apply:

1) $\Delta \omega \leq \mathrm{BW}_{\mathrm{ch}} / 2 \rightarrow H_{\mathrm{hpf}}(\Delta \omega) \approx 0 \rightarrow T(\omega) \approx 0$;

2) $A_{v}(\Delta \omega) \approx A_{v o}=$ IF amplifier DC voltage gain;

3) $A_{v o} \gg 1 \rightarrow Z_{o}(\omega) \gg Z_{\mathrm{rf}}(\omega)$.

Then the in-channel RF-to-IF gain can be simplified as

$$
A_{\mathrm{RF}-\mathrm{IF}}\left(\omega_{\mathrm{IN}}\right)=-G_{\mathrm{Ina}} R_{f} A_{\mathrm{mix}}
$$


On the other hand, the following assumptions can be made for out-of-channel interferers:

1) $\Delta \omega \gg \mathrm{BW}_{\mathrm{ch}} / 2 \rightarrow H_{\mathrm{hpf}}(\Delta \omega) \approx 1$

2) Large loop bandwidth $\rightarrow A_{v}(\Delta \omega) \gg 1 \rightarrow T(\Delta \omega) \gg 1$.

As a result, the RF-to-IF gain for out-of-channel interferers is

$$
A_{\text {RF-IF }}\left(\omega_{\text {OUT }}\right)=-\frac{G_{\text {lna }}}{G_{\text {fb }}} \frac{1}{A_{\text {mix }}}
$$

The ratio of (5) and (6) defines the maximum relative suppression of interferers due to the active feedback loop

$$
S_{\mathrm{max}}=\frac{A_{\mathrm{RF}-t o-\mathrm{IF}}\left(\omega_{\mathrm{OUT}}\right)}{A_{\mathrm{RF}-t o-\mathrm{IF}}\left(\omega_{\mathrm{IN}}\right)}=\frac{1}{G_{\mathrm{fb}} R_{f}} \frac{1}{A_{\text {mix }}^{2}}
$$

That is, to increase the relative suppression, one has to increase $R_{f}$ thereby increasing the gain of the desired signal relative to that of the interferer, and/or increase $G_{\mathrm{fb}}$ to reduce the gain of the interferer relative to that of the signal.

\section{B. RF Filter Transfer Function}

The relative suppression of interferers given by (7) is essentially the stop-band rejection of an RF channel-select filter created by the active feedback loop at the output of the LNA. Examining the filter transfer function is an alternative approach that gives further insight into the operation of the circuit.

The transfer function of the filter can be described as the normalized impedance at the output of the LNA. From (2)

$$
H_{\mathrm{ch}}(\omega)=\frac{Z_{\mathrm{CL}}(\omega)}{Z_{\mathrm{OL}}(\omega)}=\frac{1}{1+T(\omega)}
$$

The resulting filter transfer function can then be written as

$$
H_{\mathrm{ch}}(\omega)=H_{\mathrm{if}}(\Delta \omega) \cdot H_{\mathrm{rf}}(\omega)
$$

where

$$
\begin{gathered}
H_{\mathrm{if}}(\Delta \omega)=\frac{1}{1+T_{\mathrm{if}}(\Delta \omega)} \\
H_{\mathrm{rf}}(\omega)=\frac{1+j \frac{\omega}{\omega_{p}} \frac{Z_{\mathrm{rf}}(\omega)}{R_{o}+Z_{\mathrm{rf}}(\omega)}}{1+j \frac{\omega}{\omega_{p}} \frac{Z_{\mathrm{rf}}(\omega)}{R_{o}+Z_{\mathrm{rf}}(\omega)} \frac{1}{1+T_{\mathrm{if}}(\Delta \omega)}}
\end{gathered}
$$


with $\omega_{p}=1 /\left(R_{o} C_{o}\right)$ being the pole due to the output impedance of the LNA, and $T_{\text {if }}(\Delta \omega)$ is the low frequency part of the loop gain in (4), and is given by

$$
T_{\text {if }}(\Delta \omega)=G_{\mathrm{fb}} \frac{R_{o} Z_{\mathrm{if}}(\Delta \omega)}{R_{o}+Z_{\mathrm{rf}}(\omega)} A_{\text {mix }}^{2} A_{v}(\Delta \omega) H_{\mathrm{hpf}}(\Delta \omega)
$$

Thus, according to (9), the filter transfer function can be written as the product of two terms. The first term, $H_{\text {if }}(\Delta \omega)$, is the contribution of the IF part of the receiver chain up-converted around $\omega_{\mathrm{LO}}$ as evident from (3) and (12). The second term, $H_{\mathrm{rf}}(\omega)$, represents the effect of the 'RF pole' $\omega_{p}$ on the filter transfer function. Note that $H_{\mathrm{ch}}(\omega)$ approaches $H_{\mathrm{if}}(\omega)$ as $\omega_{p} \rightarrow \infty$. In fact, the dependence of $H_{\mathrm{ch}}(\omega)$ on $\omega_{p}$ is a parasitic effect that should be minimized as will be shown by the end of this section.

To gain further insight into the operation of the feedback loop and the resulting filtering effect, we start with two simplifying assumptions:

1) $\omega_{p} \gg \omega_{\mathrm{LO}} \rightarrow H_{\mathrm{ch}}(\omega) \approx H_{\mathrm{if}}(\Delta \omega)$

2) $A_{v}(\Delta \omega)=A_{v o} \gg 1 \rightarrow R_{o} \gg Z_{\mathrm{rf}}(\omega)$

The above assumptions leave the HPF as the only block determining the frequency dependence of the loop gain, mainly at small frequency offsets. Under these assumptions, substituting with (12) in (10) results in

$$
H_{\mathrm{ch}}(\omega) \approx H_{\mathrm{if}}(\Delta \omega)=\frac{1+j\left(\frac{\Delta \omega}{\omega_{\mathrm{hpf}}}\right)}{1+j\left(\frac{\Delta \omega}{\omega_{\mathrm{hpf}} /\left(1+T_{o}\right)}\right)}
$$

where $T_{o}=G_{\mathrm{fb}} R_{f} A_{\mathrm{mix}}^{2} A_{\mathrm{vo}}$ is the loop gain at $\Delta \omega=0$ with no HPF present in the loop.

The expression in (13) reveals one of the key aspects of the active feedback receiver, in which the action of the loop in shaping the transfer function from the input to the output of the receiver is to introduce a pole and a zero separated by a factor of $\left(1+T_{o}\right)$. That is, as shown in the bode plot in Fig. 5(a), the channel bandwidth is determined by the corner frequency of the HPF divided by the $\left(1+T_{o}\right)$. Thus, for a fixed resistance value of the HPF, the capacitance needed to achieve a given channel bandwidth is reduced by a factor proportional to the available loop gain. Since the major part of the loop is at IF, it is relatively easy to achieve a high loop gain and, therefore, significantly reduce the amount of capacitance/die area required. This offers a significant advantage over passive mixer filters since the capacitance values usually needed for channel selection at RF are quite large (hundreds of picofarads) as explained in section II.

To address the stability of the loop and its effect on filter characteristics, the above simplifying assumptions need to be re-examined. Towards this end, the loop can be conceptually divided 


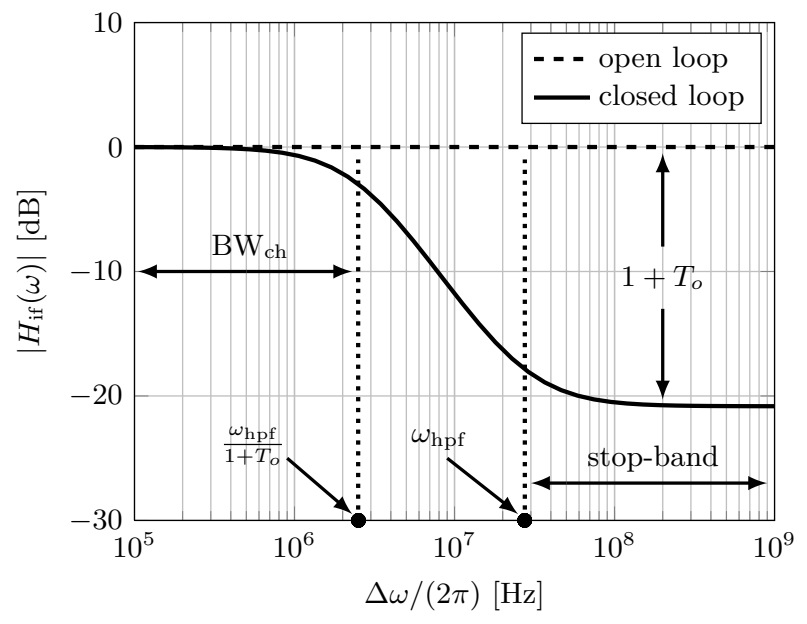

(a)

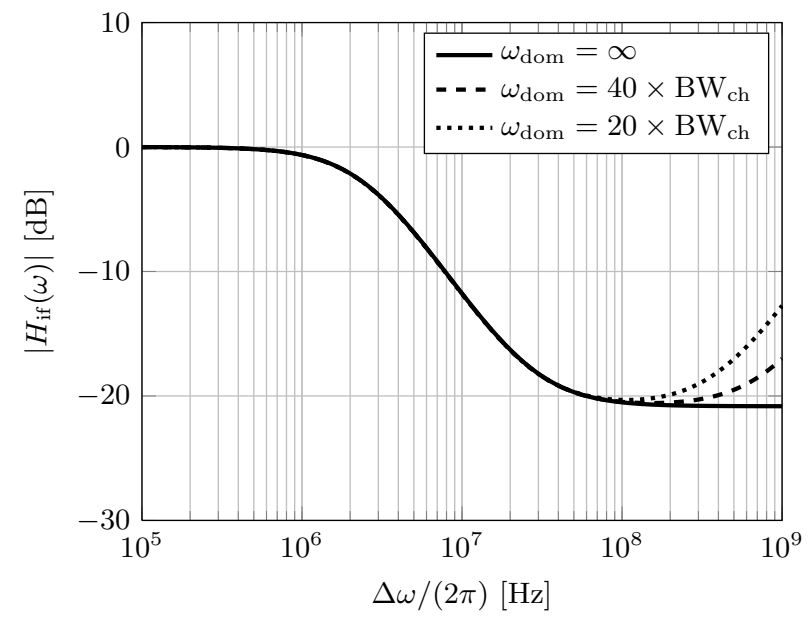

(b)

Fig. 5: Effect of IF part of the loop on the filter transfer function as given by (9) for $\omega_{p} \gg \omega_{\mathrm{LO}}, \mathrm{BW}$ ch $=5 \mathrm{MHz}(2 \times 2.5 \mathrm{MHz})$ and $T_{o}=20 \mathrm{~dB}$. (a) With infinite loop bandwidth. (b) With limited loop bandwidth determined by a dominant pole $\omega_{\text {dom }}$.

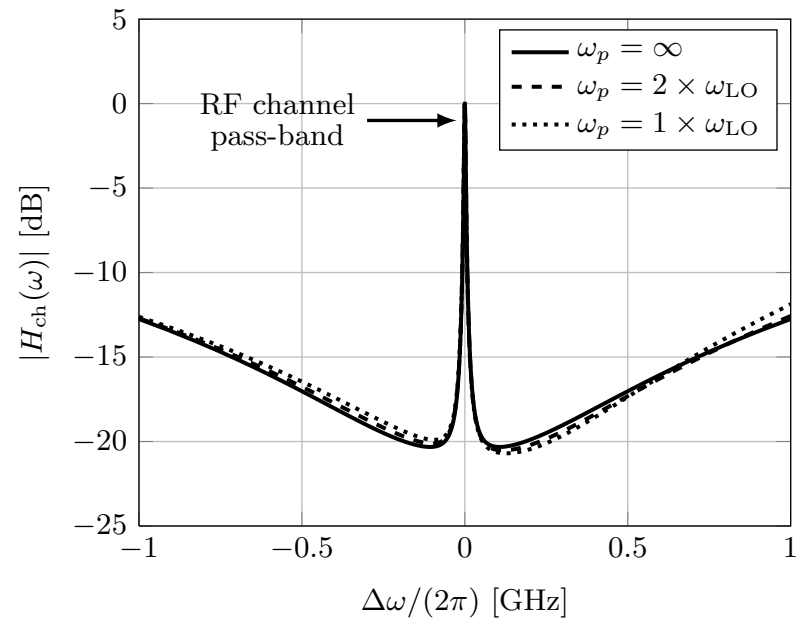

(a)

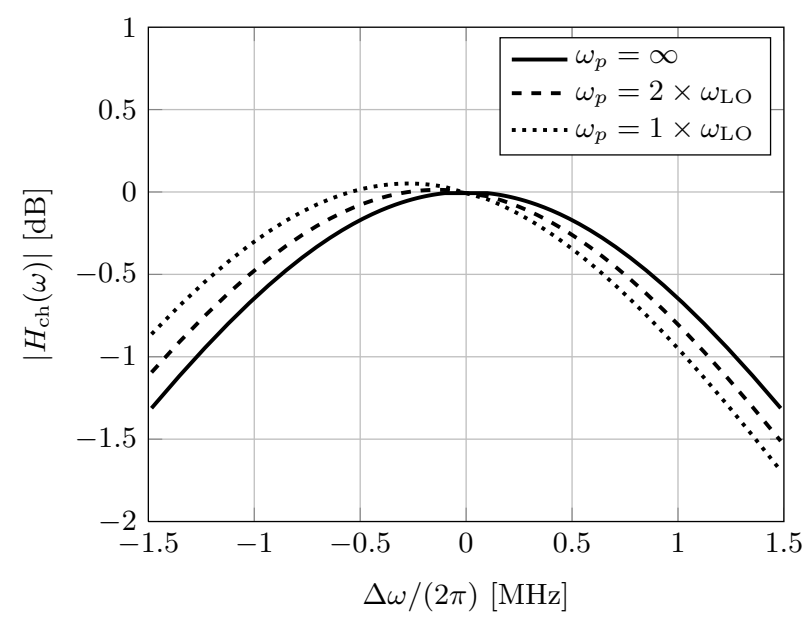

(b)

Fig. 6: Effect of RF part of the loop on the filter transfer function as given by (9) for $\omega_{\text {dom }}=20 \times \mathrm{BW}_{\mathrm{ch}}, \mathrm{BW}_{\mathrm{ch}}=5 \mathrm{MHz}$ $(2 \times 2.5 \mathrm{MHz})$ and $T_{o}=20 \mathrm{~dB}$. (a) Complete filter characteristics. (b) Zoomed-in pass-band. 
into three parts based on the frequency of operation as shown in Fig. 4: an RF part $H_{\mathrm{rf}}(\omega)$ represented by the pole $\omega_{p}$, a frequency translation interface provided by the mixers, and an IF part $H_{\text {if }}(\Delta \omega)$ representing all IF blocks.

By first examining the frequency translation interface and the IF part, one can notice that since the mixers are driven by the same LO signal, the process of down-conversion and subsequent up-conversion ideally introduces no phase shift around the loop. In other words, provided that the two mixers and their driving networks are properly matched, the phase shift around the loop is primarily relative to the frequency offset $\Delta \omega$ due to IF blocks rather than absolute frequency $\omega$ due to the mixers. Consequently, the frequency dependence of the loop gain due to both the frequency translation interface and the IF part can be introduced by adding a dominant pole $\omega_{\text {dom }}$ to the voltage gain in the transimpedance amplifier $A_{v}(\Delta \omega)$ in (4). This sets an upper limit on the loop bandwidth, or equivalently, the stop-band of the channel select filter (Fig. 5(b)). Such a filtering loop is therefore suitable for implementation in a modern high speed process and its bandwidth is expected to improve with technology scaling.

Conversely, the RF part of the loop introduces a phase shift relative to absolute frequency. As expected from (9), Fig. 6(a) shows that the asymmetric response of the RF part introduces some asymmetry in the filter shape. Furthermore, a slower RF part also results in the undesirable effect of shifting the center frequency of the filter (Fig. 6(b)). However, the IF part remains to be the dominating factor in determining the filter shape, thus, the center frequency of the filter retains programmability through changing the LO frequency.

\section{Design Example}

The results of section IV-A and IV-B can be used as a preliminary design guide. As an example, consider the following requirements:

1) Channel $\mathrm{BW}=5 \mathrm{MHz}$

2) Desired signal gain $=30 \mathrm{~dB}$

3) Interference suppression $>20 \mathrm{~dB}$

To design an active feedback receiver that meets the above specifications, we basically need to find five design parameters: $G_{\mathrm{lna}}, G_{\mathrm{fb}}, R_{f}, \omega_{\mathrm{hpf}}$ and $\omega_{\mathrm{dom}}$. Towards this end, we use the following values:

1) $A_{v o}=20 \mathrm{~dB}$ 
2) $A_{\text {mix }}=\sqrt{2} / \pi$ (double balanced design, 4 -phase $25 \%$ duty cycle LO)

3) $R_{\mathrm{SW}}=25 \Omega$

4) $R_{o}=10 \times R_{\mathrm{SW}}$

5) $\omega_{p}=1 \times \omega_{\mathrm{LO}}$

Since the required channel bandwidth is determined by the corner frequency of the HPF divided by the available loop gain (Eq. (13) and Fig. 5(a)), the corner frequency of the HPF can first be found by noting that we need a loop gain that is, at least, as large as the desired interference suppression. The design then proceeds by utilizing (5) and (6) to find the ratio of $G_{\mathrm{fb}} / G_{\text {Ina }}{ }^{1}$. Given this ratio, the actual values of $G_{\text {Ina }}, G_{\mathrm{fb}}$ and $R_{f}$ are eventually determined by the the noise requirements of the system. Finally, from (4), the stability of the loop can be guaranteed by setting the value of $\omega_{\text {dom }}$.

Based on the above procedure, Fig. 7 shows the calculated RF-to-IF gain of the designed system and the corresponding loop gain. The desired performance parameters are met with a phase margin of approximately $50^{\circ}$.

\section{NOISE AND LINEARITY}

\section{A. Noise Performance}

The noise performance of the receiver can be intuitively examined by considering the noise contributions of the forward and feedback paths separately.

The forward path is basically similar to traditional front-end receivers that have been extensively analyzed in part or as a whole in literature [10], [16]. The noise contribution of the forward path can be reduced by increasing the transconductance of the LNA and by ensuring that the switches operate with non-overlapping clocks. However, in the context of an active feedback receiver, two main issues are of specific concern; namely noise/speed requirements of the IF part of the receiver and impedance matching at the antenna interface.

In a typical receiver, the IF part, by definition, needs only to handle the down-converted channel. Thus, when considering the fundamental trade-off between noise and bandwidth, noise requirements usually take precedence. It is therefore common, even in high speed applications, to

\footnotetext{
${ }^{1}$ In case of distortion canceling (section V-B), the ratio $G_{\mathrm{fb}} / G_{\text {Ina }}$ is fixed to unity, which couples the signal gain and interference suppression requirements. This effectively creates a trade-off between in-channel and out-of-channel linearity.
} 


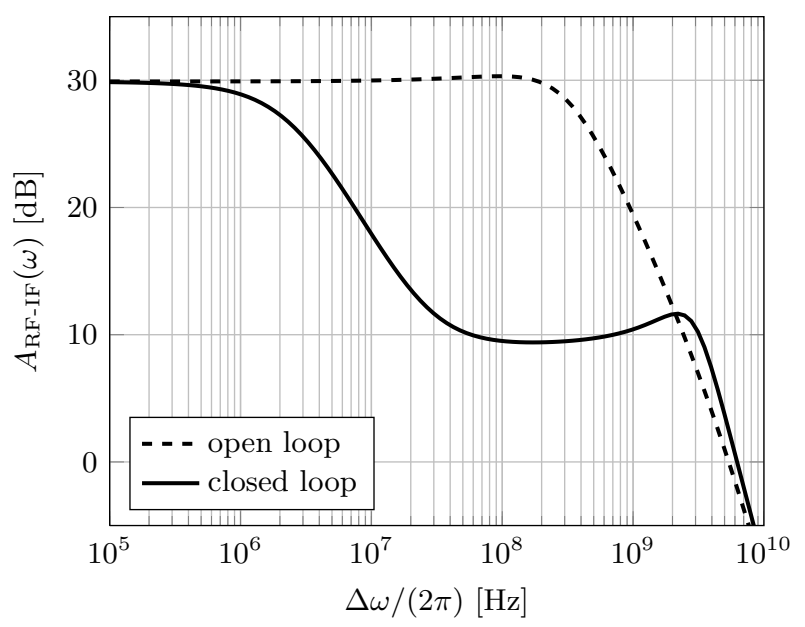

(a)

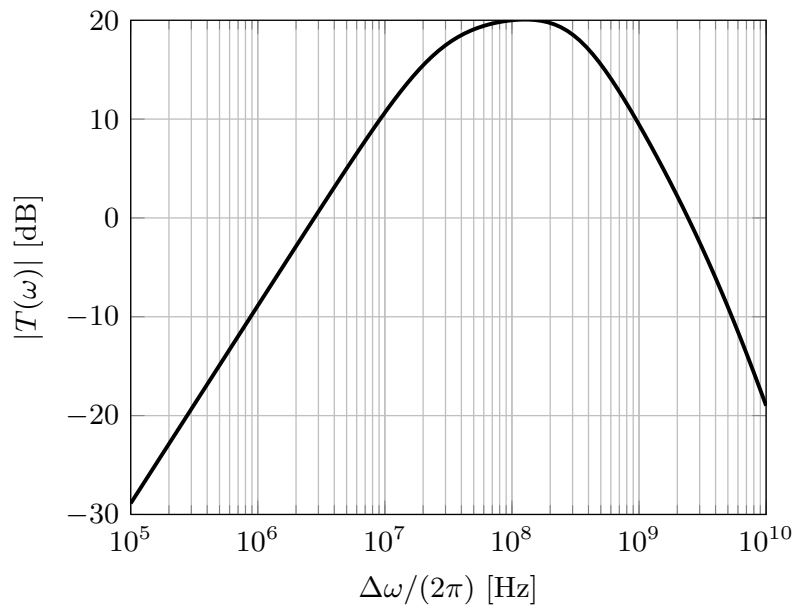

(b)

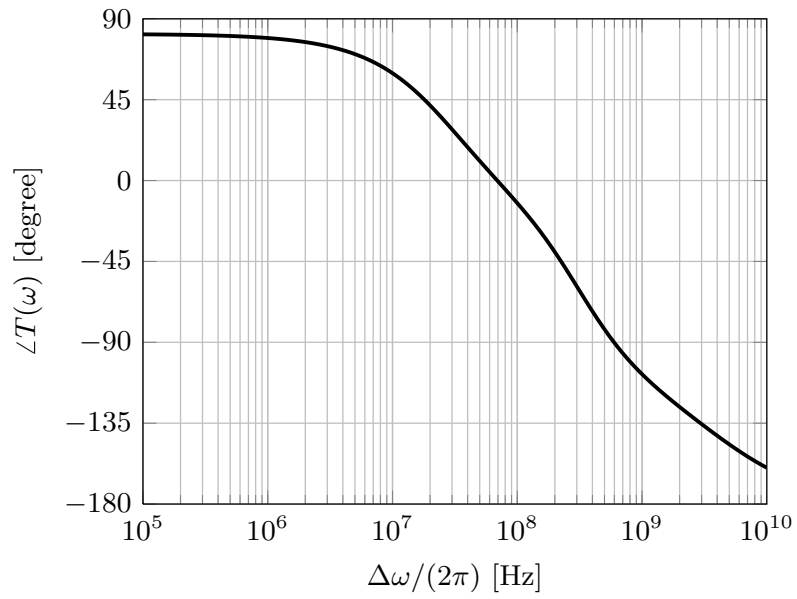

(c)

Fig. 7: Design example based on the expressions in (5), (6) and (13). Design parameters: $G_{\mathrm{Ina}}=90.7 \mathrm{mS}, G_{\mathrm{fb}}=63.7 \mathrm{mS}$, $R_{f}=1 \mathrm{k} \Omega, \omega_{\mathrm{hpf}}=2 \pi \times 27.5 \times 10^{6}$ and $\omega_{\mathrm{dom}}=2 \pi \times 50 \times 10^{6}$ 
design IF blocks that operate in weak inversion to exploit the intrinsically higher transconductance efficiency $\left(g_{m} / I\right)$ of a MOS device [12]. On the other hand, in our closed loop receiver, the bandwidth of the IF part determines the loop bandwidth, and, consequently, the stop-band of the RF filter. This suggests that the noise-speed trade-off is more pronounced in such a closed loop system. However, with the advances in CMOS scaling, weak inversion operation provides increasingly higher unity gain frequencies, well into the gigahertz range, while maintaining high $g_{m} / I$ values [17], [18]. Thus, a noise-speed trade-off is still possible, and the trade-off is expected to relax with continued technology scaling.

The entire chip prototype presented in sections VI and VII is based on self-biased inverters and switches, which, in addition to its simplicity, offers several advantages. These include a high Gain-Bandwidth product due to current re-use in inverters, eliminating the need for bias circuitry, lower second-order distortion [19], and a design that lends itself to easy porting from one technology to another. However since the inverters are biased at roughly half the supply voltage to maximize headroom and provide the same common mode level for cascading stages, they can only operate in strong inversion. As such, the aforementioned noise speed trade-off is not fully exploited.

Alternatively, a self-biased inverter structure like the one presented in [20] can be used to provide the possibility of weak/moderate inversion operation, while retaining the benefits of a simple self-biased inverter. In addition, the "improved inverter" provides roughly twice the voltage gain and reduces flicker noise due to the presence of degeneration devices for self-bias. Indeed, circuit simulations that we have been carrying out lately in a standard $65 \mathrm{~nm}$ CMOS process show that such an improved inverter is capable of achieving the same noise figure (NF) as that of a simple self-biased inverter, and lower than half its $1 /$ corner frequency, for less than one-fourth the current consumption, while still providing a gigahertz range of operation.

In addition, providing the necessary impedance match at the input of the receiver has a significant impact on its NF. Inductor based techniques such as inductive degeneration are widely used to achieve low noise $50 \Omega$ matching [21]-[23]. However, when aiming for a wide tuning range and a compact design, the use of such techniques is undesirable due to their inherent narrowband nature and the large die area required for on-chip inductors. As such, techniques like resistive matching [24], [25] or noise canceling [26], [27] present a better alternative.

For sake of simplicity to demonstrate the feedback loop, our chip prototype uses a simple $50 \Omega$ 
termination to provide input matching at the expense of $3 \mathrm{~dB}$ degradation in NF. Alternatively, a significant improvement in NF can be achieved by employing resistive shunt-feedback [24], [25]. To preserve the down-conversion operation of current commutating mixers, a two stage LNA would be required, in which the first stage provides a $50 \Omega$ match via resistive shunt-feedback, while the second stage provides a high impedance output (such an LNA can still be implemented with self-biased inverters). Another alternative is to replace the $50 \Omega$ resistor at the input of the receiver with a separate matching self-biased inverter connected in shunt with the signal path.

In the feedback path, two cases are considered as shown in Fig. 8. In case 1, the feedback transconductor is placed at IF followed by the HPF. This is a favorable arrangement since the noise of the feedback transconductor is now filtered out (together with the desired signal) before being up-converted by the mixer to the filter pass-band. This way, the active feedback loop suppresses out-of-channel interferers without introducing noise in the desired channel. Although filtering prior to the IF transconductor offers better distortion canceling as will be explained in the following section, simulations show that the NF of the receiver can degrade by as much as $10 \mathrm{~dB}$ due to the up-conversion of transconductor's flicker noise to the pass-band of the filter.

In case 2, the feedback transconductor is placed at RF which introduces one extra ' $R F$ pole' inside the loop and changes the up-conversion mixer into a voltage mixer. Since now the feedback transconductor adds only thermal noise to the channel band, its contribution to the total NF of the receiver can still be adequately suppressed by the LNA.

\section{B. Distortion Analysis}

In a conventional feedback system with high loop gain, the closed loop gain is primarily determined by the feedback ratio. Therefore, non-linearities in the feedback path are critical in determining the overall linearity of the system [28]. Thus, for a wide-band front-end receiver, utilizing a conventional feedback approach prohibits the use of active feedback components. Even if such a receiver is feasible, perfectly linear amplification of interferers would still pose strict linearity requirements on later stages in the receiver chain.

However, when employing active feedback to provide channel selection, only the desired channel appears amplified at the output while out-of-channel interferers are suppressed. Thus, although the feedback path contains non-linear elements, in principle it only needs to handle the desired signal which is typically small in amplitude. Furthermore, the linearity of later stages is 
now less of a concern due to suppressing interferers.

To quantitatively examine the overall linearity of the active feedback receiver, the different sources of distortion are considered as shown in Fig. 8 for the same two feedback cases examined in section $\mathrm{V}$-A. The circuit is then solved according to the procedure outlined in Appendix A. In its most general form, the procedure is based on obtaining the $n$-th order non-linear response of the circuit based on all $n-1$ lower order responses. However, since the receiver provides a narrow-band response for channel selection, we only focus here on third order intermodulation (IM3) products which always fall in-channel. By applying the same simplifications for in-channel

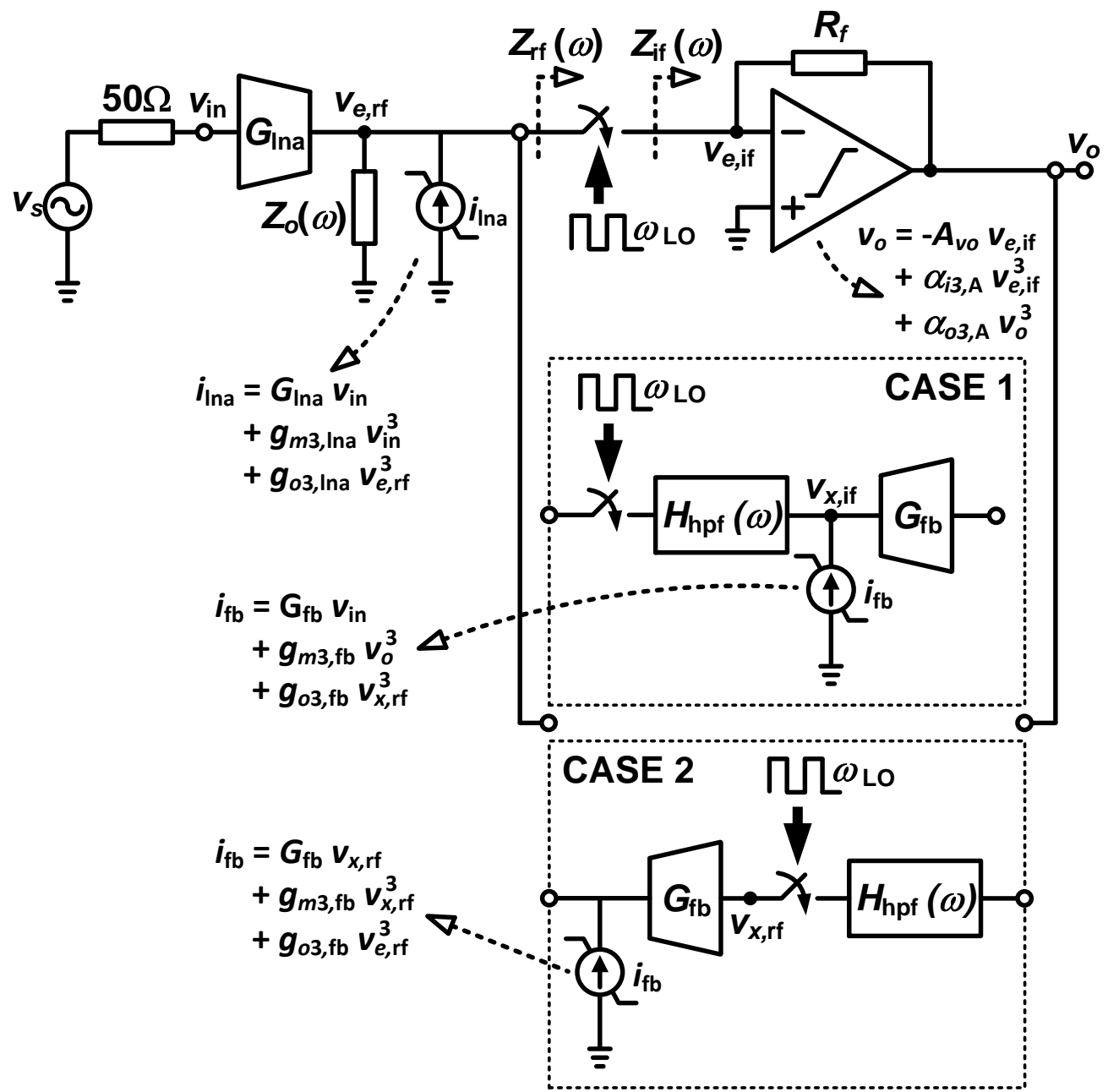

Fig. 8: Possible feedback arrangements and third order non-linear contributions in the active feedback receiver.

and out-of-channel signals used in section IV-A to the results in Appendix A, the different IM3 contributions at the output of the receiver can be summarized as shown in Table I. 
As a first observation, one can clearly see that the RF filtering provided by the active feedback loop reduces the distortion caused by out-of-channel interferers in the IF part of the receiver $\left(\alpha_{i 3, \mathrm{~A}}\right.$ and $\left.\alpha_{o 3, \mathrm{~A}}\right)$.

Furthermore, the distortion caused by in-channel interferers due to the output linearity of the LNA $\left(g_{o 3, \text { Ina }}\right)$ is reduced by the low input impedance of the transimpedance amplifier, but the reduction is limited by the switch resistance of the down-conversion mixer. This is expected since the switch resistance appears in series with the input of the transimpedance amplifier as given by (3), thus limiting the reduction in voltage swing at the output of the LNA. On the other hand, reducing the distortion of $g_{o 3, \text { lna }}$ due to out-of-channel interferers faces no such limitation. This is because, as long as the output impedance of the feedback transconductor is significantly higher than the switch resistance, providing more loop gain causes the feedback loop to sink more current independently of the switch resistance. That is, unlike passive mixer filters, filter rejection at the RF side is practically not limited by the on-resistance of the mixer switches. This has a twofold benefit. First, it enables the use of smaller switches, which directly translates to smaller switch capacitances and lower power consumption in the driving LO buffers. Second, in a modern CMOS process where output non-linearity is a major contributor to the total distortion of an active device [29], the distortion of the LNA due to out-of-channel interferers can be significantly reduced.

The most interesting observation though can be made by examining case 2 for out-of-channel interferers. One can see that the distortion products due to the V-to-I non-linearity of the LNA and feedback transconductors $\left(g_{m 3, \text { Ina }}\right.$ and $g_{m 3, \text { fb }}$, respectively) have opposite polarities. Such a direct consequence of the feedback action suggests that some form of distortion canceling is possible. This can be examined by considering only these two distortion components and expressing the change in IM3 distortion due to closed loop operation as

$$
\begin{aligned}
\Delta \mathrm{IM} 3 & =\frac{\mathrm{IM} 3_{g m 3, \text { Ina }}+\mathrm{IM} 3_{g m 3, \mathrm{fb}}}{\mathrm{IM} 3_{g m 3, \text { nna }}} \\
& =\frac{g_{m 3, \text { nna }}-g_{m 3, \mathrm{fb}}\left(G_{\text {Ina }} / G_{\mathrm{fb}}\right)^{3}}{g_{m 3, \text { nna }}}
\end{aligned}
$$

Since $G_{\mathrm{fb}} / G_{\mathrm{Ina}}=g_{m 3, \mathrm{fb}} / g_{m 3 \text {, Ina }}$, denoting this ratio as $m$ and re-arranging (14) yields the simple relation

$$
\Delta \mathrm{IM} 3=1-\frac{1}{m^{2}}
$$


By plotting (15) in Fig. 9, one can distinguish two regions of operation separated by the border case of $m=1$. For $m<1$, closed loop operation actually causes an increase in IM3 distortion as $m$ is reduced. This is expected since the RF-to-IF gain of out-of-channel interferers is inversely proportional to $m$ as given by (6), which means that the feedback path needs to handle an amplified version of the interferers present at the input of the receiver, causing its distortion to dominate over that of the LNA. On the other hand, when increasing $m$ beyond unity, the change in IM3 distortion approaches zero since now the feedback path needs to handle an attenuated version of the interferers and the distortion of the LNA determines the linearity performance. Increasing $m$ also corresponds to a higher loop gain which improves the linearity of the IF part of the receiver as discussed earlier. Thus, although Fig. 9 only considers $g_{m 3, \operatorname{lna}}$ and $g_{m 3, \mathrm{fb}}$, the region for $m>1$ also shows that the non-linearity of the LNA, which is outside the loop, sets an upper limit on the linearity of the whole receiver chain as expected. Note that this insight into the operation of the loop is in agreement with the general discussion at the beginning of this section.

However, Fig. 9 shows that this limitation can be overcome by setting $m$ equal to unity, i.e. by matching the LNA and feedback transconductances. Under this condition, the distortion of the LNA can be canceled because an inverted replica of input interferers is forced at the output via the feedback action, causing the feedback transconductance to perfectly sink the distortion currents sourced by the LNA. In other words, with the aid of negative feedback, the LNA and the feedback transconductor form a voltage mirror for out-of-channel interferers. This arrangement has several advantages:

1) It cancels LNA distortion caused by out-of-channel interferers without placing the LNA inside the loop, thus avoiding injecting noise and mixer harmonics at the antenna, which significantly decouples the noise-linearity-harmonic radiation trade-off that typically exists in passive mixer filters (section II).

2) As opposed to having the LNA inside the loop, filtering at the output of the LNA eliminates at least one extra pole from the loop, which results in a higher loop BW for the same phase margin.

3) It is a large signal linearization based on matching a non-linear V-to-I operation with a similar non-linear I-to-V operation.

4) It is based on feedback and matching transconductances, which makes it robust to process 
spread.

5) The non-linearity of the feedback path is not a limitation.

\begin{tabular}{|c|c|c|c|c|}
\hline \multirow[b]{2}{*}{ Non-linearity } & \multicolumn{2}{|c|}{ Case 1} & \multicolumn{2}{|c|}{ Case 2} \\
\hline & $\begin{array}{l}\text { IM3 product due to } \\
\text { in-channel interferers }\end{array}$ & $\begin{array}{l}\text { IM3 product due to } \\
\text { out-of-channel interferers }\end{array}$ & $\begin{array}{l}\text { IM3 product due to } \\
\text { in-channel interferers }\end{array}$ & $\begin{array}{c}\text { IM3 product due to } \\
\text { out-of-channel interferers }\end{array}$ \\
\hline$g_{m 3, \operatorname{lna}}$ & $\begin{array}{c}-\frac{3}{4} g_{m 3, \operatorname{lna}} A^{3} \\
\cdot R_{f} A_{\text {mix }}\end{array}$ & $\begin{array}{c}-\frac{3}{4} g_{m 3, \operatorname{lna}} A^{3} \\
\cdot R_{f} A_{\text {mix }}\end{array}$ & $\begin{array}{c}-\frac{3}{4} g_{m 3, \operatorname{lna}} A^{3} \\
\cdot R_{f} A_{\text {mix }}\end{array}$ & $\begin{array}{c}-\frac{3}{4} g_{m 3, \operatorname{lna}} A^{3} \\
\quad \cdot R_{f} A_{\text {mix }}\end{array}$ \\
\hline$g_{o 3, \operatorname{lna}}$ & $\begin{array}{c}-\frac{3}{4} g_{o 3, \operatorname{lna}} A^{3} \\
\cdot R_{f} A_{\text {mix }} \\
G_{\operatorname{lna}}^{3}\left(R_{\mathrm{SW}}+\frac{R_{f}}{A_{v o}}\right)^{3}\end{array}$ & $\begin{array}{c}-\frac{3}{4} g_{o 3, \ln a} A^{3} \\
\cdot R_{f} A_{\text {mix }} \\
\cdot\left(\frac{G_{\text {Ina }}}{G_{\mathrm{fb}}}\right)^{3}\left(\frac{1}{A_{\text {mix }}^{2} A_{v o}}\right)^{3}\end{array}$ & $\begin{array}{c}-\frac{3}{4} g_{o 3, \operatorname{lna}} A^{3} \\
\cdot R_{f} A_{\text {mix }} \\
\cdot G_{\text {Ina }}^{3}\left(R_{\mathrm{SW}}+\frac{R_{f}}{A_{v o}}\right)^{3}\end{array}$ & $\begin{array}{c}-\frac{3}{4} g_{o 3, \operatorname{lna}} A^{3} \\
\cdot R_{f} A_{\text {mix }} \\
\cdot\left(\frac{G_{\text {ma }}}{G_{\mathrm{fb}}}\right)^{3}\left(\frac{1}{A_{\text {mix }}^{2} A_{v o}}\right)^{3}\end{array}$ \\
\hline$\alpha_{i 3, \mathrm{~A}}$ & $\begin{array}{c}+\frac{3}{4} \alpha_{i 3, \mathrm{~A}} A^{3} \\
\cdot\left(G_{\operatorname{lna}} \frac{R_{f}}{A_{v o}} A_{\text {mix }}\right)^{3}\end{array}$ & $\begin{array}{c}+\frac{3}{4} \alpha_{i 3, \mathrm{~A}} A^{3} \\
\cdot\left(\frac{G_{\text {lna }}}{G_{\mathrm{fb}}}\right)^{3}\left(\frac{1}{A_{\text {mix }} A_{v o}}\right)^{3}\end{array}$ & $\begin{array}{c}+\frac{3}{4} \alpha_{i 3, \mathrm{~A}} A^{3} \\
\cdot\left(G_{\operatorname{lna}} \frac{R_{f}}{A_{v o}} A_{\text {mix }}\right)^{3}\end{array}$ & $\begin{array}{c}+\frac{3}{4} \alpha_{i 3, \mathrm{~A}} A^{3} \\
\cdot\left(\frac{G_{\text {lna }}}{G_{\mathrm{fb}}}\right)^{3}\left(\frac{1}{A_{\text {mix }} A_{v o}}\right)^{3}\end{array}$ \\
\hline$\alpha_{o 3, \mathrm{~A}}$ & $\begin{array}{c}-\frac{3}{4} \alpha_{o 3, \mathrm{~A}} A^{3} \\
\cdot\left(G_{\operatorname{lna}} R_{f} A_{\text {mix }}\right)^{3}\end{array}$ & $\begin{array}{c}-\frac{3}{4} \alpha_{o 3, \mathrm{~A}} A^{3} \\
\cdot\left(\frac{G_{\text {Ina }}}{G_{\text {fb }}}\right)^{3}\left(\frac{1}{A_{\text {mix }}}\right)^{3}\end{array}$ & $\begin{array}{c}-\frac{3}{4} \alpha_{o 3, \mathrm{~A}} A^{3} \\
\cdot\left(G_{\operatorname{lna}} R_{f} A_{\text {mix }}\right)^{3}\end{array}$ & $\begin{array}{c}-\frac{3}{4} \alpha_{o 3, \mathrm{~A}} A^{3} \\
\cdot\left(\frac{G_{\mathrm{max}}}{G_{\mathrm{fb}}}\right)^{3}\left(\frac{1}{A_{\operatorname{mix}}}\right)^{3}\end{array}$ \\
\hline$g_{m 3, \mathrm{fb}}$ & 0 & 0 & 0 & $\begin{array}{c}+\frac{3}{4} g_{m 3, \mathrm{fb}} A^{3} \\
\cdot R_{f} A_{\text {mix }}\left(\frac{G_{\mathrm{Ina}}}{G_{\mathrm{fb}}}\right)^{3}\end{array}$ \\
\hline$g_{o 3, \mathrm{fb}}$ & 0 & 0 & $\begin{array}{c}-\frac{3}{4} g_{o 3, \mathrm{fb}} A^{3} \\
\cdot R_{f} A_{\text {mix }} \\
\cdot G_{\text {Ina }}^{3}\left(R_{\mathrm{SW}}+\frac{R_{f}}{A_{v o}}\right)^{3}\end{array}$ & $\begin{array}{c}-\frac{3}{4} g_{o 3, \mathrm{fb}} A^{3} \\
\cdot R_{f} A_{\text {mix }} \\
\cdot\left(\frac{G_{\mathrm{lna}}}{G_{\mathrm{fb}}}\right)^{3}\left(\frac{1}{A_{\text {mix }}^{2} A_{v o}}\right)^{3}\end{array}$ \\
\hline
\end{tabular}

TABLE I: Third order intermodulation distortion products in an active feedback receiver.

In case 1, our simplified analysis predicts that such cancellation is not possible due to the presence of the HPF between the two transconductors which prevent the in-channel IM3 product from flowing between the two transconductors. However, with the up-conversion mixer placed between the two transconductors, full circuit simulations of the receiver show that partial cancellation is still possible. The cancellation in this case still exhibits the same dependence on the ratio of transconductors (i.e. maximum partial cancellation for equal transconductors), but, due to the frequency translation between the inputs of two transconductors, only holds when the BW of the LNA is higher than the loop bandwidth plus LO frequency. Further investigation of 


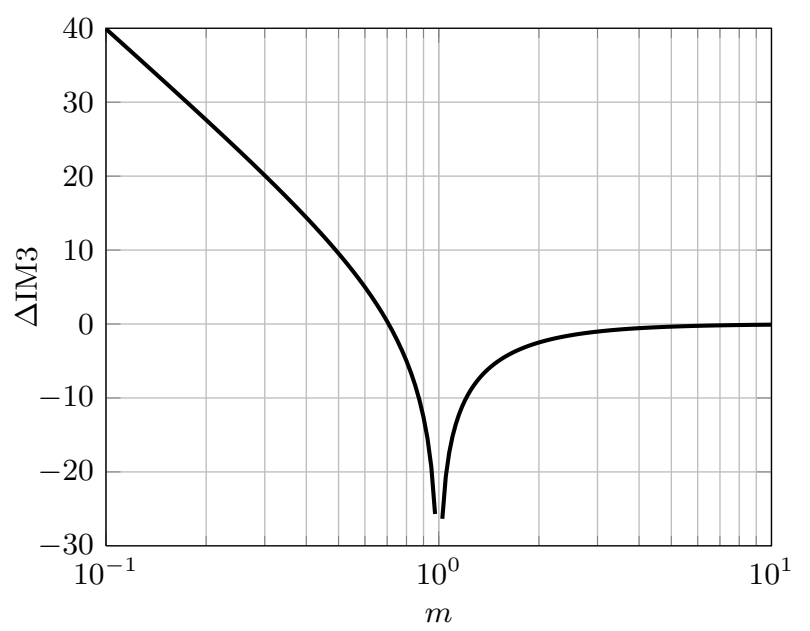

Fig. 9: Change in out-of-channel IM3 due to $g_{m 3, \text { Ina }}$ and $g_{m 3, \mathrm{fb}}$ as a function of the ratio of feedback and LNA transconductances $m$ (case 2 in Table I).

these observations is still required.

\section{IMPLEMENTATION}

To verify the concept, an active feedback receiver front-end was designed and implemented in a $65 \mathrm{~nm}$ low power (LP) standard CMOS process. As explained in section V-A, the design shown in Fig. 10 is entirely based on self-biased inverters and switches.

One set of switches arranged in a double balanced fashion down-converts the differential RF input (i.e. 2-phase real signal) into differential I/Q signals (i.e. 4-phase complex signal) at IF. A similar set of switches in the feedback path combines the I/Q signals to perform the reverse operation. As expected, the $25 \Omega$ switches are about 3-to-5 times smaller than what is typically seen in state-of-the-art designs using passive mixers for filtering [7], [8]. The feedback transconductors are placed at IF and followed by the HPF to filter out flicker noise before being up-converted to the channel band. Furthermore, the LNA and feedback transconductors are matched to exploit partial IM3 cancellation as explained in section V-B. Although placing the matched feedback transconductors at RF would, in general, offer better cancellation, the relatively high output impedance of the forward path in this design severely degrades the loop gain when driving the voltage mixers necessary for up-conversion. Therefore, partial IM3 cancellation is chosen as a compromise in this design. 
An on-chip clock divider is included to generate the 4-phase LO necessary for differential I/Q operation. The measurement interface provides isolation to measure the output without disturbing the loop. Noise and full channel bandwidth can be measured via the inverter buffers, while actual in-band gain and linearity can be measured via large $(15 \mathrm{k} \Omega)$ output resistors.

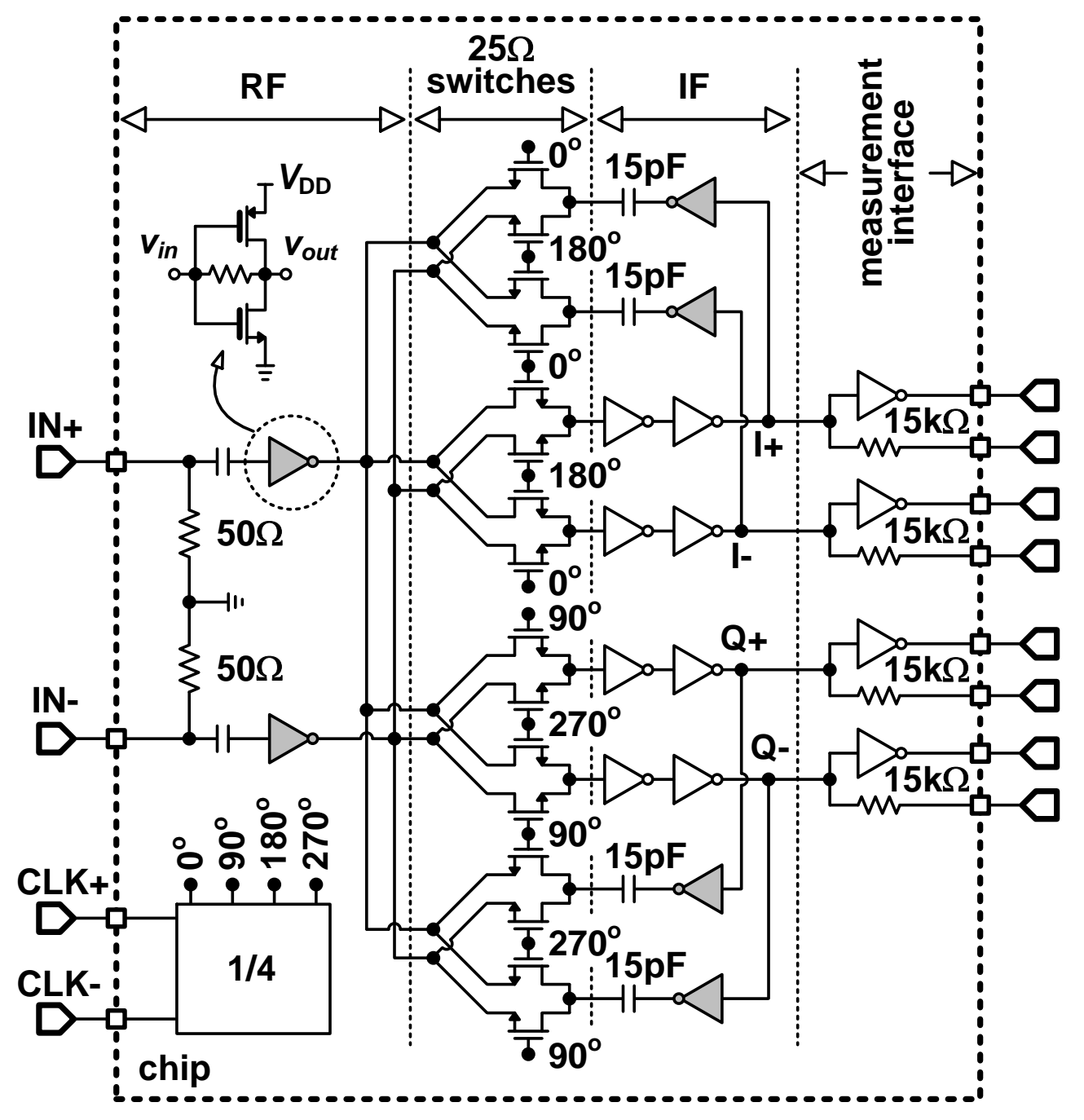

Fig. 10: Schematic of implemented active feedback receiver.

The feedback loop in the designed receiver has a gain of about $20 \mathrm{~dB}(\approx 10 \times$ reduction in capacitance) and a unity gain frequency of $>500 \mathrm{MHz}$ on either side of the LO frequency. The total capacitance in this design is only $60 \mathrm{pF}(15 \mathrm{pF} \times 4$ for differential I/Q operation) to achieve a $5 \mathrm{MHz}$ channel bandwidth. The benefit in area reduction is evident from the die photograph in Fig. 11. The chip is pad limited due to the multiple outputs required for testing, with an active 


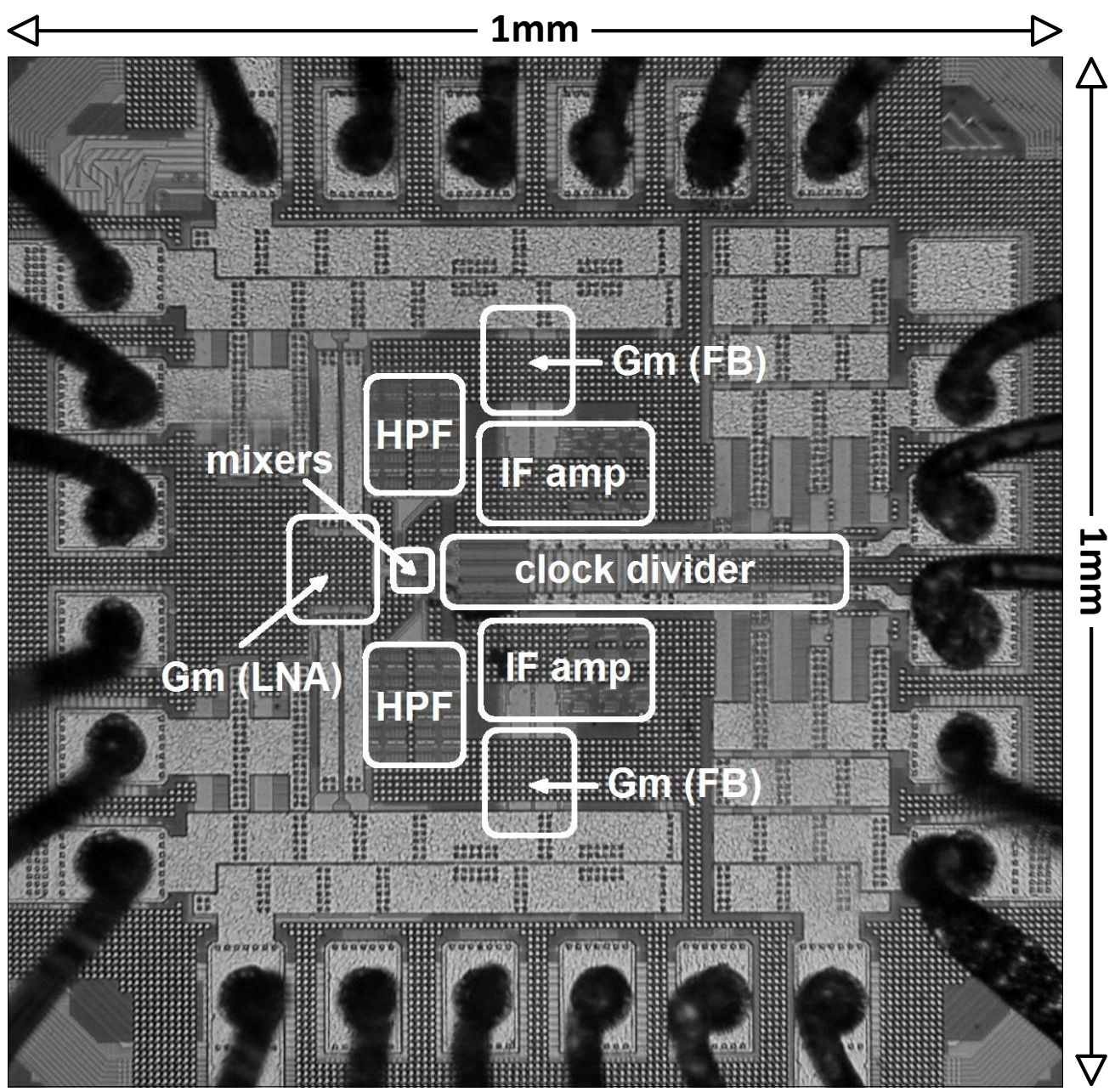

Fig. 11: Die photograph.

area $<0.06 \mathrm{~mm}^{2}$ including the clock circuitry.

\section{Simulation And Measurement Results}

Table II shows a comparison of simulated key receiver parameters for open and closed loop operation. The closed loop in-band gain drops by less than $4 \mathrm{~dB}$ due to the loading effect of the feedback path. As expected the NF degradation due to closed loop operation is minor (0.3dB) because the noise of the feedback path within the desired channel bandwidth is heavily filtered out by the HPF. At a relatively small frequency offset of $20 \mathrm{MHz}$, the receiver rejection ratio is improved by almost $14 \mathrm{~dB}$, achieved at RF at the output of the LNA.

Consequently, as expected, two-tone simulations show an improvement in maximum wideband IIP3 of about $15 \mathrm{~dB}$, achieved at more than 3 times lower frequency offset compared to the open 
loop case. Similar improvement is simulated for the wideband blocking performance of the receiver, i.e. the blocker power level at which the small signal gain of the desired signal drops by $1 \mathrm{~dB}(\mathrm{P}-1 \mathrm{~dB})$. The current consumption in closed loop operation is higher by approximately $20 \mathrm{~mA}$ mainly due to the four IF transconductors matched to the LNA.

Since filtering after the LNA would normally cause the LNA to set an upper limit on the overall linearity of the receiver (section V-B), the improvement in IIP3 due to distortion canceling is examined by comparing the maximum IIP3 achieved by the active feedback receiver to the maximum IIP3 that can be achieved by the LNA. Simulations of the self-biased inverter LNA with $50 \Omega$ matching at its input and the same test tones listed in Table II show a maximum IIP3 of approximately $4 \mathrm{dBm}$, achieved when the LNA output is presented with a perfect AC short circuit. Comparing this value to that listed in Table II indicates that the effect of distortion canceling is about 8 -to-9dB.

\begin{tabular}{|c|c|c|c|}
\hline & open loop & closed loop & \\
\hline \hline RF-to-IF gain $(\Delta f=50 \mathrm{kHz})$ & 34.8 & 31 & $\mathrm{~dB}$ \\
\hline DSB NF $(\Delta f=2 \mathrm{MHz})$ & 7.2 & 7.5 & $\mathrm{~dB}$ \\
\hline Receiver rejection $(\Delta f=20 \mathrm{MHz})$ & 1.9 & 15.5 & $\mathrm{~dB}$ \\
\hline Maximum wideband IIP3 & -2.3 & 12.1 & $\mathrm{dBm}$ \\
\hline Maximum wideband P-1dB & -16.2 & -6 & $\mathrm{dBm}$ \\
\hline Current consumption & $3 f=500 \mathrm{MHz}$ & $\Delta f=160 \mathrm{MHz}$ & \\
\hline \multicolumn{2}{|c|}{$3 f .5$} & 50.1 & $\mathrm{~mA}$ \\
\hline
\end{tabular}

TABLE II: Simulated key parameters of implemented active feedback receiver for open and closed loop operation for $f_{\mathrm{LO}}=2 \mathrm{GHz}$. For two-tone measurements, tones are located at $f_{\mathrm{LO}}+\Delta f$ and $f_{\mathrm{LO}}+2 \Delta f-25 \mathrm{kHz}$. For blocking performance measurements, the desired signal and blocker are located at $f_{\mathrm{LO}}+50 \mathrm{kHz}$ and $f_{\mathrm{LO}}+\Delta f$, respectively.

Figure 12(a) shows the measured RF-to-IF gain for positive and negative frequency offsets $(\Delta f)$ for the case of a $2 \mathrm{GHz} \mathrm{LO}$ frequency $\left(f_{\mathrm{LO}}\right)$. The receiver achieves a gain of $30 \mathrm{~dB}$ (measurement buffers de-embedded), and a channel bandwidth of $5 \mathrm{MHz}(2.5 \mathrm{MHz}$ on either side of the LO). A maximum stop-band rejection of $48 \mathrm{~dB}$ is achieved at $250 \mathrm{MHz}$ frequency 
offset. The variation between positive and negative frequency offsets is $\pm 0.5 \mathrm{~dB}$ across the entire range of measured frequency offsets. The measured RF-to-IF gain for $\Delta f>0$ and different values of $f_{\mathrm{LO}}$ is also shown in Fig. 12(b), with a similar variation range of $\pm 0.5 \mathrm{~dB}$. Similar results have been measured for $\Delta f<0$. According to the analysis in section IV-B, such a symmetry of the measured RF-to-IF gain for positive and negative frequency offsets, as well as the tunability for different values of LO frequency, indicate that the operation of the loop is primarily determined by the IF blocks and that the bandwidth of the RF part of the loop is not a limitation.

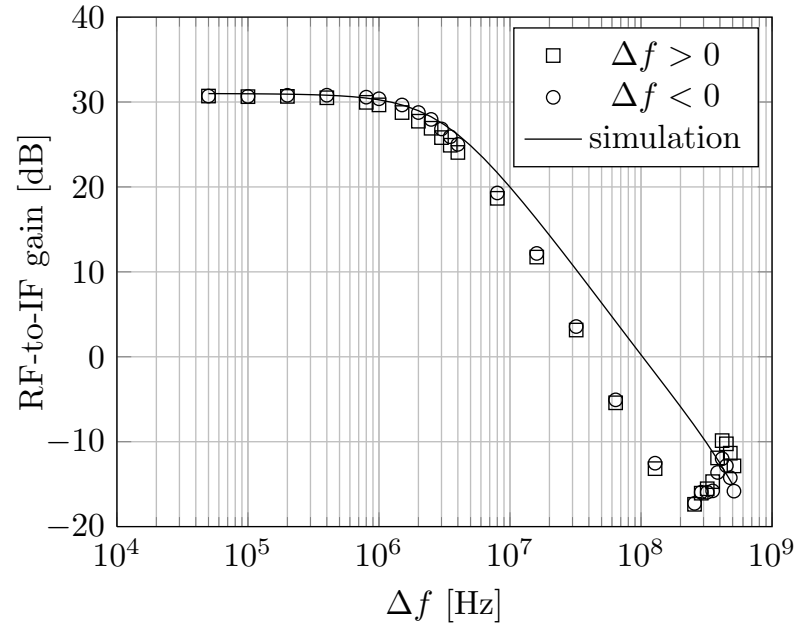

(a)

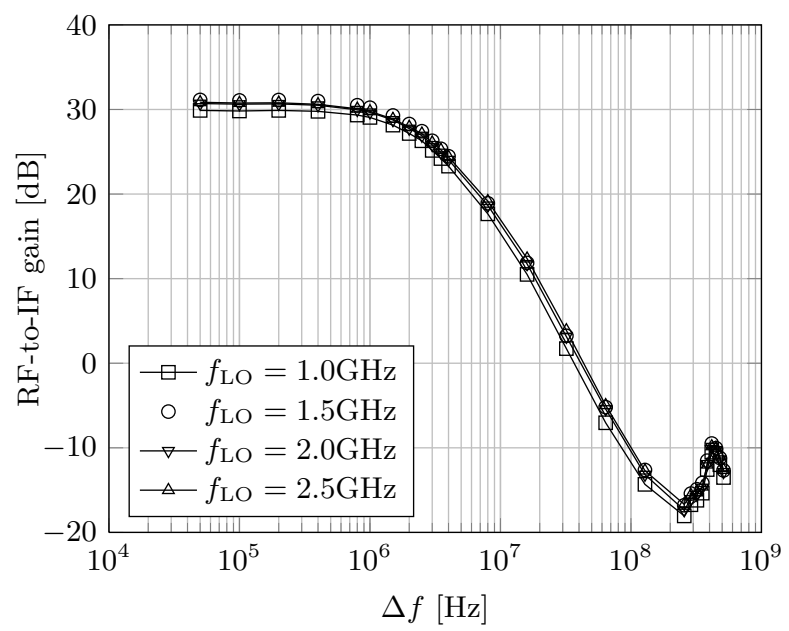

(b)

Fig. 12: Measured RF-to-IF gain. (a) For positive and negative frequency offsets and $f_{\mathrm{LO}}=2 \mathrm{GHz}$. Solid line indicates circuit simulation. Similar results have been measured (and simulated) for $f_{\mathrm{LO}}=1,1.5$ and $2.5 \mathrm{GHz}$. (b) For $\Delta f>0$ and different values of $f_{\mathrm{LO}}$. Similar results have been measured for $\Delta f<0$.

The RF-to-IF gain has also been measured for 10 chip samples. The results for $f_{\mathrm{LO}}=2 \mathrm{GHz}$ and $\Delta f>0$ are shown in Fig. 13. The average RF-to-IF gain is $30.8 \mathrm{~dB}$ and the average maximum stop-band rejection is $48.2 \mathrm{~dB}$ (Fig. 13(a)). The standard deviation of gain versus frequency offset is shown in Fig. 13(b), with a maximum in-band deviation of around 0.6dB at $\Delta f=2.5 \mathrm{MHz}$, and a maximum stop-band rejection deviation of less than $1.6 \mathrm{~dB}$ at $\Delta f=$ $300 \mathrm{MHz}$. The corresponding standard deviation of channel bandwidth for $\Delta f>0$ is shown in Fig.13(c), with a mean channel bandwidth of $2.4 \mathrm{MHz}$ on either side of the LO. Similar results, with similar statistics, have been measured across the entire range of measurement $(-500 \mathrm{MHz} \leq$ 
$\Delta f \leq+500 \mathrm{MHz}$ for $\left.1 \mathrm{GHz} \leq f_{\mathrm{LO}} \leq 2.5 \mathrm{GHz}\right)$

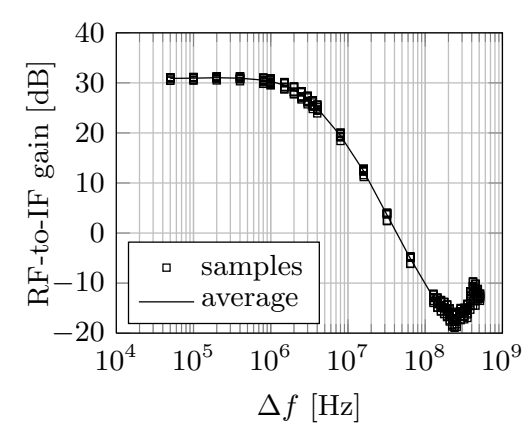

(a)

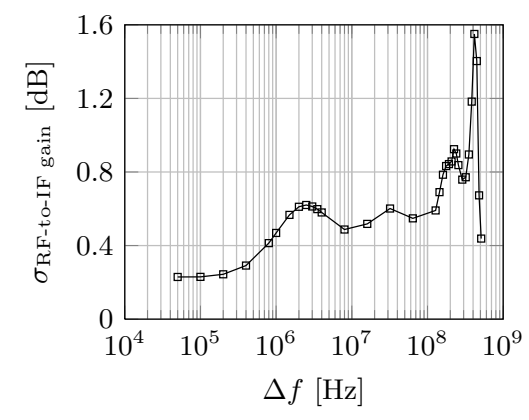

(b)

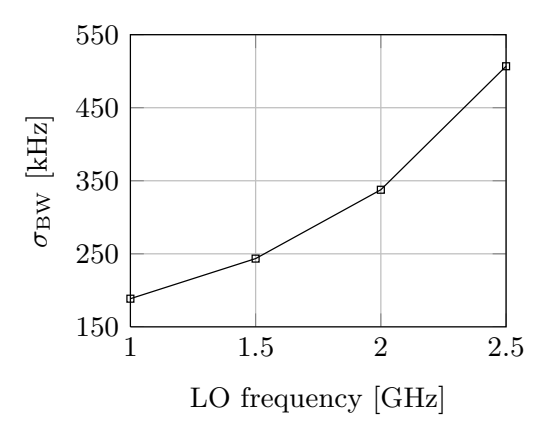

(c)

Fig. 13: Measured RF-to-IF gain for 10 chip samples for $f_{\mathrm{LO}}=2 \mathrm{GHz}$ and $\Delta f>0$. Similar results, with similar statistics, have been measured across the entire range of measurement $\left(-500 \mathrm{MHz} \leq \Delta f \leq+500 \mathrm{MHz}\right.$ for $1 \mathrm{GHz} \leq f_{\mathrm{LO}} \leq 2.5 \mathrm{GHz}$ ). (a) RF-to-IF gain. Solid line indicates the average. (b) Standard deviation of RF-to-IF gain. (c) Corresponding standard deviation of channel bandwidth around a mean value of $2.4 \mathrm{MHz}$ for $\Delta f>0$.

Fig. 14 shows a measured DSB NF in the range of $7.25-8.9 \mathrm{~dB}$ for $f_{\mathrm{LO}}=1-2.5 \mathrm{GHz}$, measured at a single differential output (I or Q) at a frequency offset of $2 \mathrm{MHz}(1 / \mathrm{f}$ noise corner frequency $=500 \mathrm{kHz}$ ). As discussed in section $\mathrm{V}-\mathrm{A}$, and as shown by the simulation results in Table II, the relatively high NF of the receiver is not due to a fundamental limitation of the active feedback architecture, but mainly due to the simple $50 \Omega$ termination at the input and the use of self-biased inverters operating in strong inversion regime. As discussed in section V-A, by employing an alternative matching technique like resistive shunt-feedback, an estimated 1.5 to $2 \mathrm{~dB}$ lower NF can be achieved. In addition, by employing the improved self-biased inverters discussed in section V-A, significant reduction in NF for the same power level becomes feasible.

The linearity of the active feedback receiver is examined with two-tone measurements. Since we are only interested in intermodulation products that fall inside the channel bandwidth, measurement is carried out with the two tones located at frequency offsets of $\Delta f$ and $2 \Delta f-25 \mathrm{kHz}$, such that the lower intermodulation product always falls in-channel at $25 \mathrm{kHz}$. The measurement results shown in Fig. 15 show a wide-band IIP3 $>+12 \mathrm{dBm}$ for interferers at $>60 \mathrm{MHz}$ offset. The difference between in-channel IIP3 (about $-20 \mathrm{dBm}$ ) and wideband IIP3 indicates an improvement of $>33 \mathrm{~dB}$ thanks mainly to the feedback loop, with 8 to $9 \mathrm{~dB}$ of that improvement 


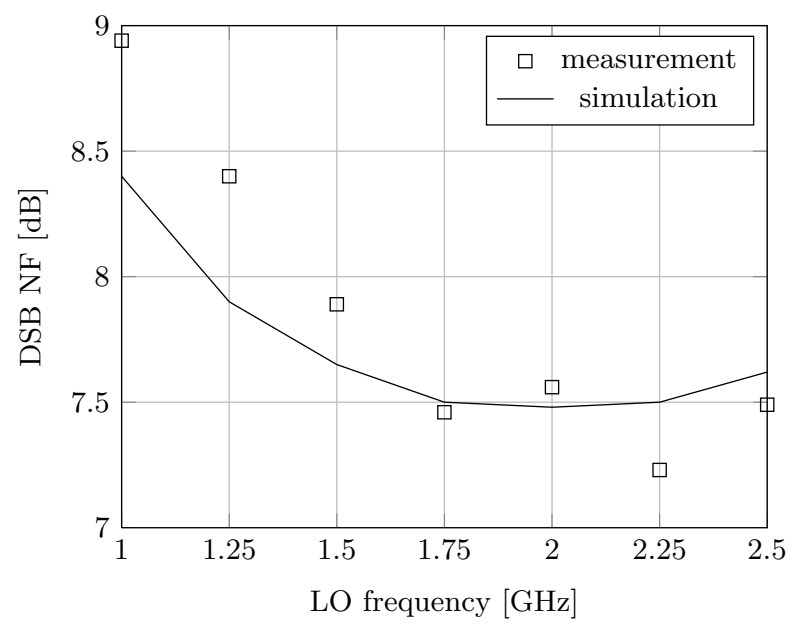

Fig. 14: Measured DSB noise figure at $\Delta f=2 \mathrm{MHz}$. Solid line indicates circuit simulation.

due to partial IM3 cancellation as indicated from simulations.

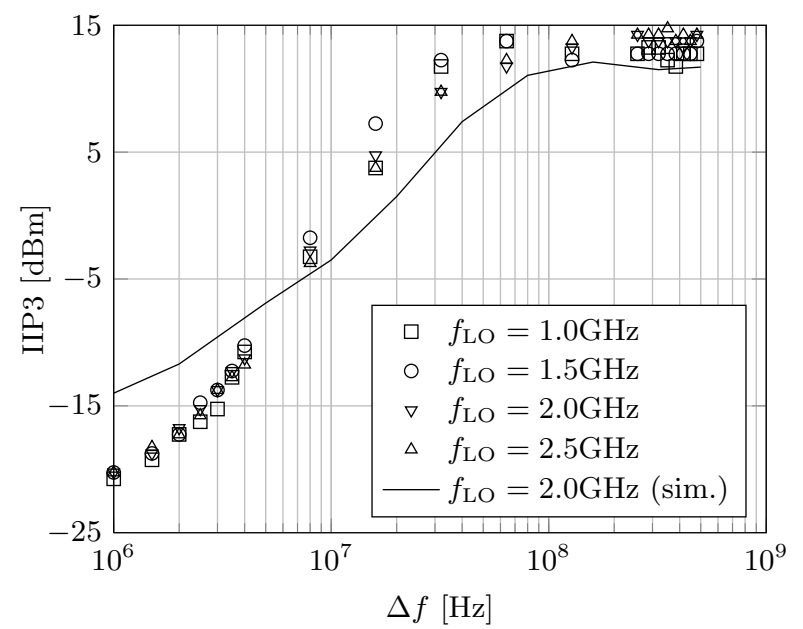

Fig. 15: Measured IIP3 for $\Delta f>0$. The two test tones are located at $f_{\mathrm{LO}}+\Delta f$ and $f_{\mathrm{LO}}+2 \Delta f-25 \mathrm{kHz}$. Solid line indicates circuit simulation for $f_{\mathrm{LO}}=2 \mathrm{GHz}$ and $\Delta f>0$. Similar results have been measured (and simulated) for $\Delta f<0$.

In addition, IIP3 measurements for 10 chip samples are shown in Fig. 16 for $f_{\mathrm{LO}}=2 \mathrm{GHz}$ and $\Delta f>0$, with the solid line indicating the average IIP3 (Fig.16(a)) and a maximum standard deviation of $1.6 \mathrm{~dB}$ (Fig.16(b)). Similar results with similar statistics have been measured for negative frequency offsets and all values of LO frequencies.

Fig. 17 shows the blocking performance of the receiver. Measured P-1dB exhibits a maximum of $-3 \mathrm{dBm}$ for a blocker at $120 \mathrm{MHz}$ ( $>19 \mathrm{~dB}$ improvement). Similarly, P-1dB measurements 


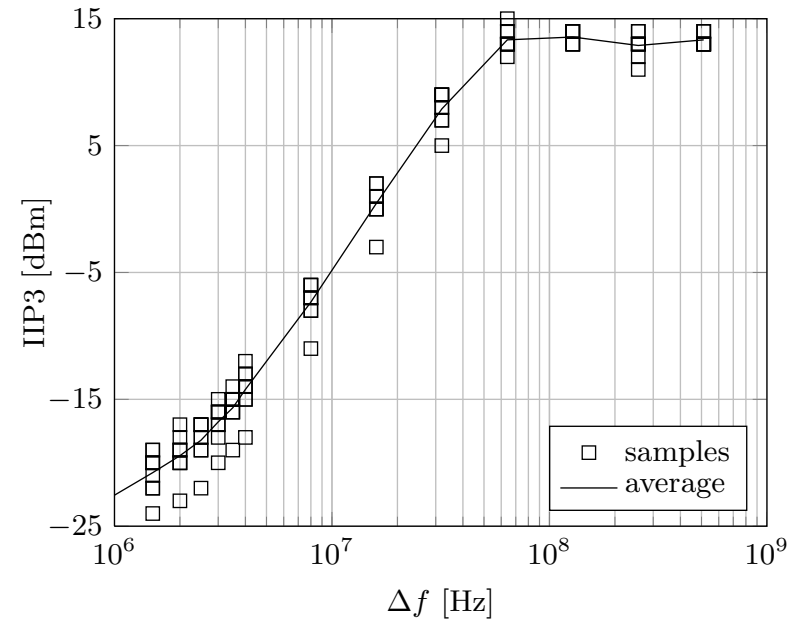

(a)

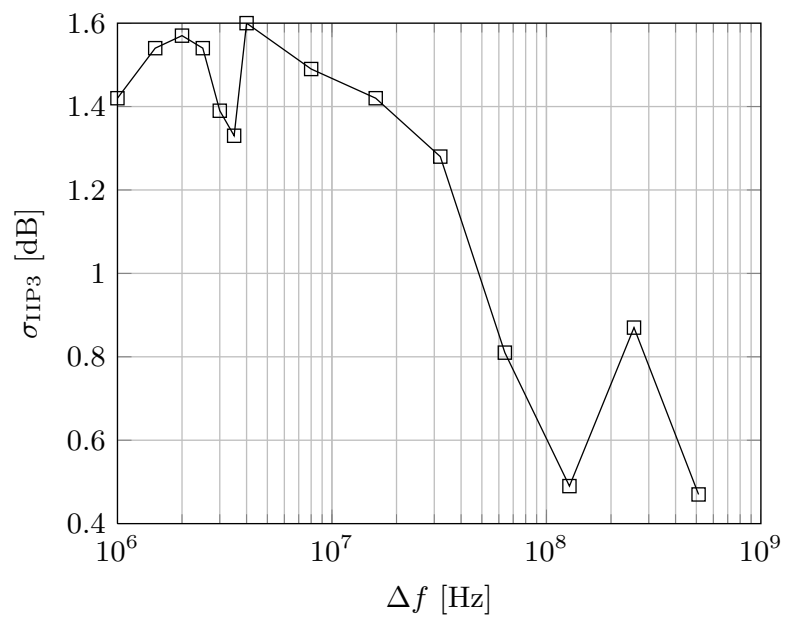

(b)

Fig. 16: Measured IIP3 for 10 chip samples for $f_{\mathrm{LO}}=2 \mathrm{GHz}$ and $\Delta f>0$. The two test tones are located at $f_{\mathrm{LO}}+\Delta f$ and $f_{\mathrm{LO}}+2 \Delta f-25 \mathrm{kHz}$. Similar results, with similar statistics, have been measured across the entire range of measurement $\left(-500 \mathrm{MHz} \leq \Delta f \leq+500 \mathrm{MHz}\right.$ for $\left.1 \mathrm{GHz} \leq f_{\mathrm{LO}} \leq 2.5 \mathrm{GHz}\right)$. (a) IIP3. Solid line indicates the average. (b) Corresponding standard deviation.

for 10 chip samples are also shown in Fig. 18 for $f_{\mathrm{LO}}=2 \mathrm{GHz}$ and $\Delta f>0$.

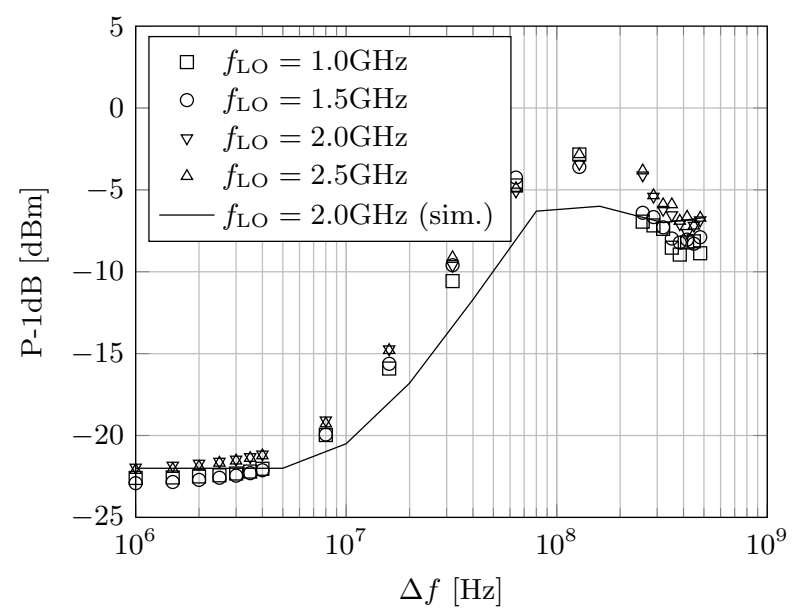

Fig. 17: Measured blocking performance. The desired signal and the blocker are located at $f_{\mathrm{LO}}+50 \mathrm{kHz}$ and $f_{\mathrm{LO}}+\Delta f$, respectively. Solid line indicates circuit simulation for $f_{\mathrm{LO}}=2 \mathrm{GHz}$ and $\Delta f>0$. Similar results have been measured (and simulated) for $\Delta f<0$.

The circuit core consumes $62 \mathrm{~mW}$ (excluding clock circuitry and measurement buffers) from 


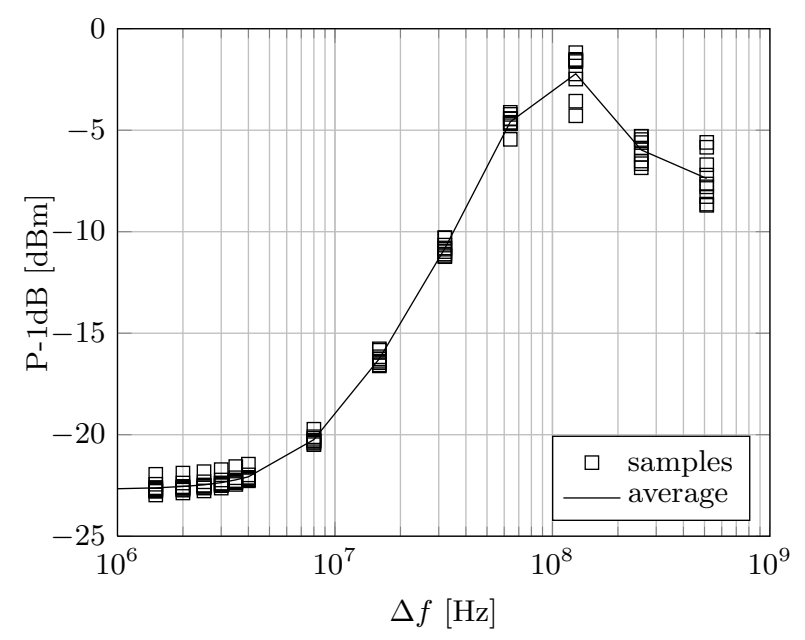

(a)

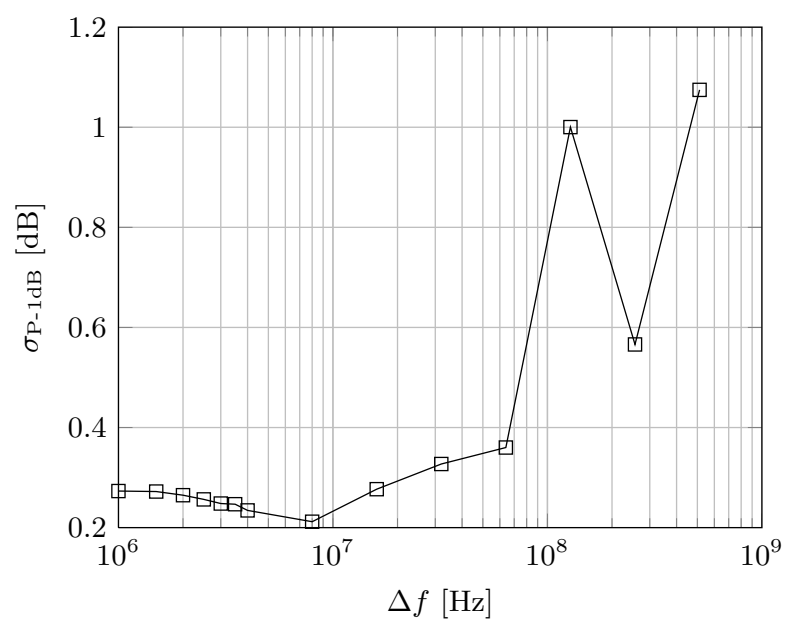

(b)

Fig. 18: Measured $\mathrm{P}-1 \mathrm{~dB}$ for 10 chip samples for $f_{\mathrm{LO}}=2 \mathrm{GHz}$ and $\Delta f>0$. The desired signal and the blocker are located at $f_{\mathrm{LO}}+50 \mathrm{kHz}$ and $f_{\mathrm{LO}}+\Delta f$, respectively. Similar results, with similar statistics, have been measured across the entire range of measurement $\left(-500 \mathrm{MHz} \leq \Delta f \leq+500 \mathrm{MHz}\right.$ for $1 \mathrm{GHz} \leq f_{\mathrm{LO}} \leq 2.5 \mathrm{GHz}$ ). (a) $\mathrm{P}-1 \mathrm{~dB}$. Solid line indicates the average. (b) Corresponding standard deviation.

\section{a $1.2 \mathrm{~V}$ supply.}

Table III gives a summary of measured parameters and compares performance to other stateof-the-art receivers. The presented design occupies about 80 to $97 \%$ less die area, while achieving comparable or better performance. The bandwidth of $5 \mathrm{MHz}$ is suitable for most applications in the 1 to $2.5 \mathrm{GHz}$ range and increasing it would further reduce the die size. Compared to the highest stop-band rejection $(\approx 50 \mathrm{~dB})$ reported by the superheterodyne architecture in [6], the direct conversion receiver presented in this paper achieves a comparable rejection of (48dB) at about 5 times the frequency offset while occupying $<8 \%$ of the die area. Better or comparable wideband IIP3 of $>+12 \mathrm{dBm}$ is measured at 2.5 to 13 times lower frequency offset $(60 \mathrm{MHz})$ compared to most reported values [12], [30]. Moreover, this design significantly outperforms previously reported feedback-based receivers [30], [31] in terms of gain, frequency range, stopband rejection and wideband IIP3 while maintaining a competitive performance for other receiver parameters. 


\begin{tabular}{|c|c|c|c|c|c|c|c|c|}
\hline & This work & [6] & [12] & [13] & [30] & [31] & [32] & \\
\hline Technique & $\begin{array}{c}\text { active } \\
\text { feedback }\end{array}$ & $\begin{array}{c}\text { super- } \\
\text { heterodyne }\end{array}$ & $\begin{array}{l}\text { harmonic } \\
\text { rejection }\end{array}$ & mixer-first & $\begin{array}{c}\text { active } \\
\text { feedback }\end{array}$ & $\begin{array}{c}\text { active } \\
\text { feedback }\end{array}$ & feedforward & \\
\hline Technology & $65 \mathrm{~nm}$ & $65 \mathrm{~nm}$ & $65 \mathrm{~nm}$ & $65 \mathrm{~nm}$ & $0.18 \mu \mathrm{m}$ & $65 \mathrm{~nm}$ & $65 \mathrm{~nm}$ & CMOS \\
\hline Active Area & $<0.06$ & 0.76 & $<1$ & 2 & $<1.8$ & 1.2 & 0.28 & $\mathrm{~mm}^{2}$ \\
\hline RF frequency & $1-2.5$ & $1.8-2$ & $<1$ & $0.1-2.4$ & 1.9 & 1.9 & 1.9 & $\mathrm{GHz}$ \\
\hline Gain & 30 & 55 & 34.4 & $40-70$ & 22.7 & 22.5 & 20.9 & $\mathrm{~dB}$ \\
\hline Channel BW & 5 & - & 12 & 20 & - & - & 4.5 & $\mathrm{MHz}$ \\
\hline DSB NF & $7.25-8.9$ & 2.8 & 4 & $<5$ & 4.1 & $<8$ & 6.8 & $\mathrm{~dB}$ \\
\hline $\begin{array}{l}\text { Stop-band } \\
\text { rejection }\end{array}$ & $\begin{array}{c}48 \\
\Delta f=250 \mathrm{MHz}\end{array}$ & $\begin{array}{c}\approx 50 \\
\Delta f=40 \mathrm{MHz}\end{array}$ & - & - & $\begin{array}{c}27 \\
\Delta f=180 \mathrm{MHz}\end{array}$ & $\begin{array}{c}10.5 \\
\Delta f=5 \mathrm{MHz}\end{array}$ & $\begin{array}{c}>21 \\
\Delta f \approx 50 \mathrm{MHz}\end{array}$ & $\mathrm{dB}$ \\
\hline $\begin{array}{c}\text { Wideband } \\
\text { IIP3 }\end{array}$ & $\begin{array}{c}>+12 \\
\Delta f=60 \mathrm{MHz}\end{array}$ & - & $\begin{array}{c}+18 \\
\Delta f=800 \mathrm{MHz}\end{array}$ & $\begin{array}{c}-8 \text { to }+27 \\
\Delta f=20 \mathrm{MHz}\end{array}$ & $\begin{array}{c}+7.5 \\
\Delta f \approx 150 \mathrm{MHz}\end{array}$ & - & - & $\mathrm{dBm}$ \\
\hline $\begin{array}{c}\text { Wideband } \\
\text { IIP2 }\end{array}$ & $\begin{array}{c}>49 \\
\Delta f>30 \mathrm{MHz}\end{array}$ & - & $\begin{array}{c}51 \\
\Delta f \approx 1.2 \mathrm{GHz}\end{array}$ & $\begin{array}{l}56 \\
-\end{array}$ & - & - & - & $\mathrm{dBm}$ \\
\hline $\begin{array}{c}\text { Wideband } \\
\text { P-1dB }\end{array}$ & $\begin{array}{c}-3 \\
\Delta f=120 \mathrm{MHz}\end{array}$ & - & $\begin{array}{c}-5 \\
\Delta f=465 \mathrm{MHz}\end{array}$ & $\begin{array}{l}-26 \text { to }+5 \\
\Delta f=40 \mathrm{MHz}\end{array}$ & $\begin{array}{c}+1 \\
\Delta f \approx 150 \mathrm{MHz}\end{array}$ & $\begin{array}{c}<-15 \\
\Delta f=20 \mathrm{MHz}\end{array}$ & $\begin{array}{c}>0 \\
\Delta f=80 \mathrm{MHz}\end{array}$ & $\mathrm{dBm}$ \\
\hline $\begin{array}{c}\text { Power } \\
\text { consumption }\end{array}$ & 62 & - & 39.6 & $<70$ & $<63$ & 375 & 72 & $\mathrm{~mW}$ \\
\hline $\begin{array}{l}\text { Supply } \\
\text { voltage }\end{array}$ & 1.2 & - & 1.2 & $1.2 / 2.5$ & 1.8 & 2.5 & $1.2 / 2.5$ & $\mathrm{~V}$ \\
\hline
\end{tabular}

TABLE III: Summary of measurement results and comparison to other state-of-the-art receivers.

\section{CONCLUSION}

In this paper, active feedback for RF channel selection has been presented. Filtering is achieved by employing a frequency translation loop with a high loop gain to convert an IF HPF to an RF channel-select filter. The large value of on-chip capacitance typically needed in passive mixer filters is reduced in the active feedback receiver by a factor proportional to the available loop gain, which results in a highly compact design. In a modern high speed CMOS process, the RF filter characteristics are primarily determined by the IF part of the loop and, thus, the filter maintains tunability through changing the clock frequency. In addition, due to the active nature of 
the feedback path, the maximum rejection achievable in the stop-band of the filter is not limited by the switch resistance of the mixers. This allows for using significantly smaller switches and consequently translates to lower power consumption in the LO buffers. Furthermore, the feedback loop offers the possibility of utilizing a voltage mirror for canceling the IM3 distortion products caused by out-of-channel interferers in the LNA. This eliminates the limitation set by the LNA on the linearity of the receiver chain without filtering prior to the LNA, therefore avoiding noise and harmonics injection at the antenna.

A prototype of a direct conversion receiver employing an active feedback loop has been designed and implemented in a $65 \mathrm{~nm}$ standard CMOS process. The design occupies 80 to $97 \%$ less die area compared to other state-of-the-art receivers and is tunable over a range of 1 to $2.5 \mathrm{GHz}$. The receiver achieves a measured maximum stop-band rejection of $48 \mathrm{~dB}$ and a measured wideband IIP3 $>+12 \mathrm{dBm}$, while maintaining a competitive performance for other receiver parameters.

\section{APPENDIX A}

\section{NON-LINEAR SYSTEM RESPONSE}

In this section, a general procedure for deriving the response of a non-linear amplifier with feedback is given and applied for the active feedback receiver.

The procedure is outlined in Fig. 19 and is as follows:

(a) Given a small signal excitation $v_{s}$, the first order (linear) response of the system is first obtained. The first order response is the set of all node voltages (or, equivalently, branch currents).

(b) The excitation source is then de-activated and the first order voltages are used as inputs to find the second order response of the system.

(c) In a similar manner, the third order response can be obtained by considering the sum of first and second order voltages as inputs to a third order system.

(d) The procedure is repeated for higher order responses.

Strictly speaking, the above procedure is an approximation because it ignores further interactions between the resulting distortion components. For instance, the third order distortion products obtained in step 3 mix with first and second order voltages via the second order term $a_{2}$. 
However, such secondary effects render the procedure quite cumbersome for hand calculations while providing no insight and marginal accuracy benefits.

The aforementioned procedure can now be applied to the active feedback system shown in Fig. 8 where only third order non-linearities are considered. The aim is to find the third order intermodulation (IM3) response of the system in response to two-tone excitation.

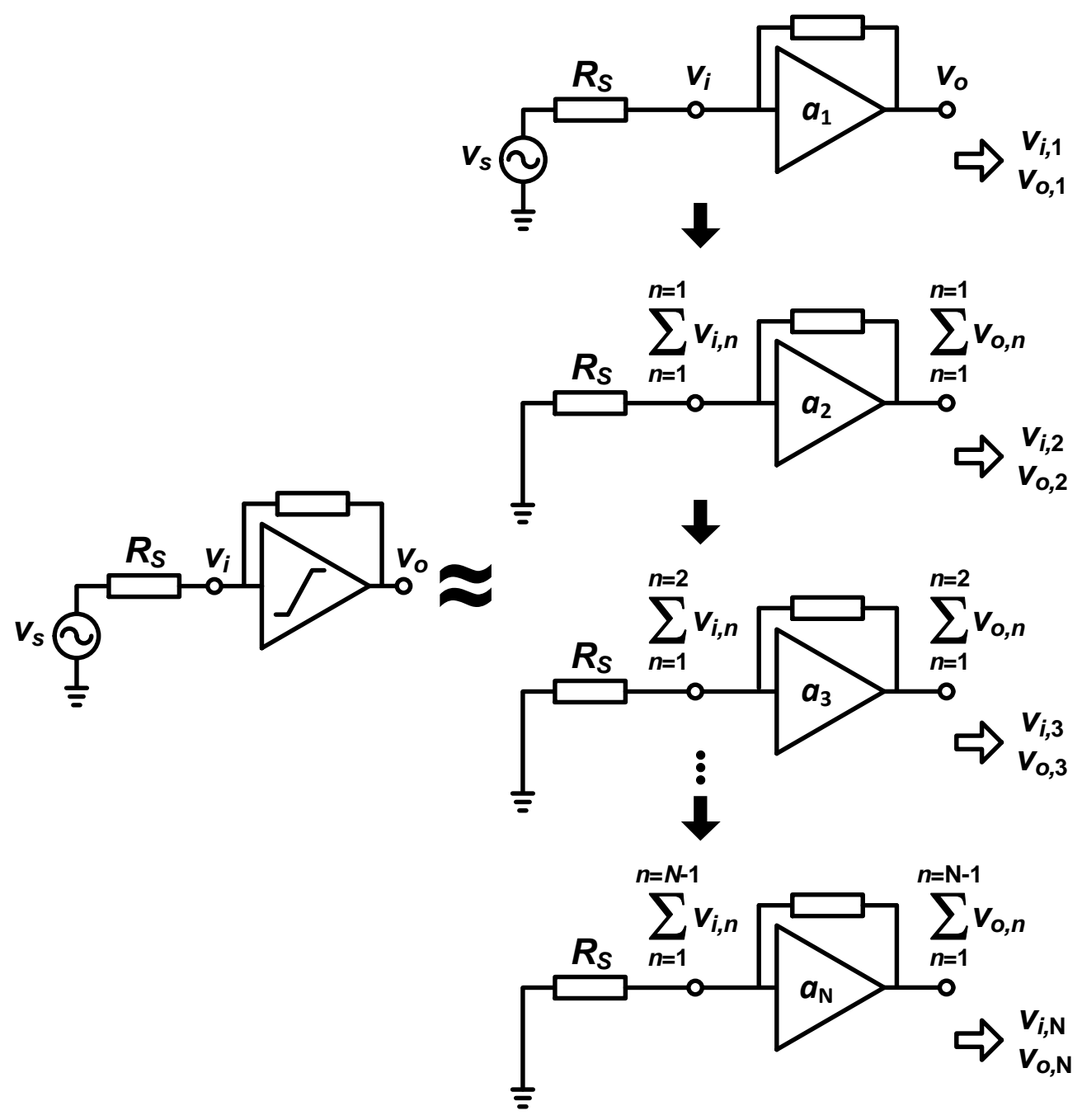

Fig. 19: Non-linear analysis procedure.

By first examining the transfer function of the system derived in section IV-A, the first order node voltages of the forward path can be written as

$$
v_{o 1}=-G_{\mathrm{ln}} Z_{\mathrm{CL} 1} A_{\mathrm{mix}} A_{v 1} v_{\mathrm{in} 1}
$$




$$
\begin{gathered}
v_{e, \mathrm{if} 1}=G_{\mathrm{lna}} Z_{\mathrm{OL} 1} A_{\mathrm{mix}} v_{\mathrm{in} 1}+G_{\mathrm{fb}} Z_{\mathrm{OL} 1} A_{\mathrm{mix}}^{2} H_{\mathrm{hpf} 1} v_{o 1} \\
v_{e, \mathrm{rf} 1}=\left(A_{\mathrm{mix}}+\frac{R_{\mathrm{SW}}}{A_{\mathrm{mix}} Z_{\mathrm{if} 1}}\right) v_{e, \mathrm{if} 1}
\end{gathered}
$$

where the shorthand subscript notation is used to indicate the frequency of interest, i.e. $X_{1}=$ $X\left(\Delta \omega_{1}\right)$ for IF quantities and $X_{1}=X\left(\omega_{\mathrm{LO}}+\Delta \omega_{1}\right)$ for RF quantities, where $X$ is any circuit quantity with frequency dependence.

In addition, approximating the HPF as a reciprocal block, the first order node voltages of the feedback path can be found

$$
\begin{gathered}
v_{x, \mathrm{if} 1}=A_{\mathrm{mix}} H_{\mathrm{hpf} 1} v_{e, \mathrm{ff} 1} \\
v_{x, \mathrm{rf} 1}=A_{\mathrm{mix}} H_{\mathrm{hpf} 1} v_{o 1}
\end{gathered}
$$

Having determined the first order response, we can now proceed with finding the desired third order response.

\section{A. IM3 Distortion due to the Forward Path}

In the forward path, the following non-linear contributions are considered:

(a) Third order LNA non-linearity due to input excitation $\left(g_{m 3, \ln a}\left[\mathrm{I} / \mathrm{V}^{3}\right]\right)$.

(b) Third order LNA non-linearity due to output excitation $\left(g_{o 3, \ln a}\left[\mathrm{I} / \mathrm{V}^{3}\right]\right)$.

(c) Third order opamp non-linearity due to input excitation $\left(\alpha_{i 3, \mathrm{~A}}\left[\mathrm{~V} / \mathrm{V}^{3}\right]\right)$.

(d) Third order opamp non-linearity due to output excitation $\left(\alpha_{o 3, \mathrm{~A}}\right)\left[\mathrm{V} / \mathrm{V}^{3}\right]$.

Based on an input two-tone excitation of amplitude $A$ and the first order voltages in (16)-(18), the IM3 output voltage for each of the non-linear coefficients listed above can be written as

$$
\begin{gathered}
v_{o 3 a}=-\frac{3}{4} g_{m 3, \ln a} Z_{\mathrm{OL} 3} A_{\mathrm{mix}} A_{v 3} A^{3}-T_{3} v_{o 3 a} \\
v_{o 3 b}=-\frac{3}{4} g_{o 3, \ln a} Z_{\mathrm{OL} 3} A_{\mathrm{mix}} A_{v 3} v_{e, \mathrm{rf} 1}^{3}-T_{3} v_{o 3 b} \\
v_{o 3 c}=+\frac{3}{4} \alpha_{i 3, \mathrm{~A}} v_{e, \mathrm{if} 1}^{3}-T_{3} v_{o 3 c} \\
v_{o 3 d}=+\frac{3}{4} \alpha_{o 3, \mathrm{~A}} v_{o 1}^{3}-T_{3} v_{o 3 d}
\end{gathered}
$$

where again the shorthand subscript notation is used for the IM3 response i.e. $X_{3}=X\left(\Delta \omega_{\mathrm{IM} 3}\right)$ for IF quantities and $X_{3}=X\left(\omega_{\mathrm{LO}}+\Delta \omega_{\mathrm{IM} 3}\right)$ for RF quantities, where $X$ is any circuit quantity with frequency dependence and $\Delta \omega_{\mathrm{IM} 3}$ is the frequency offset of the IM3 product. 
The first term on the right-hand side of (21)-(24) represents the third order intermodulation distortion product generated by the LNA, while the second term in each of the equations represents the linear feedback of the IM3 product through the active feedback loop. Substituting with (16)-(18) in (21)-(24) and collecting terms yields the required IM3 distortion products

$$
\begin{gathered}
v_{o 3 a}=-\frac{3}{4} g_{m 3, \ln a} Z_{\mathrm{CL} 3} A_{\text {mix }} A_{v 3} A^{3} \\
v_{o 3 b}=-\frac{3}{4} g_{o 3, \operatorname{lna}} Z_{\mathrm{CL} 3} A_{\text {mix }} A_{v 3}\left(G_{\mathrm{lna}} \frac{Z_{o 1} \| Z_{\mathrm{rf} 1}}{1+T_{1}}\right)^{3} A^{3} \\
v_{o 3 c}=+\frac{3}{4} \alpha_{i 3, \mathrm{~A}} \frac{1}{1+T_{3}}\left(G_{\mathrm{lna}} Z_{\mathrm{CL} 1} A_{\text {mix }}\right)^{3} A^{3} \\
v_{o 3 d}=-\frac{3}{4} \alpha_{o 3, \mathrm{~A}} \frac{1}{1+T_{3}}\left(G_{\mathrm{lna}} Z_{\mathrm{CL} 1} A_{\mathrm{mix}} A_{v 1}\right)^{3} A^{3}
\end{gathered}
$$

\section{B. Distortion due to the Feedback Path}

In the feedback path, the following non-linear contributions are considered for each of the cases shown in Fig. 8:

(a) Case 1: Third order non-linearity of the IF feedback transconductance due to input excitation $\left(g_{m 3, \mathrm{fb}}\left[\mathrm{I} / \mathrm{V}^{3}\right]\right)$.

(b) Case 1: Third order non-linearity of the IF feedback transconductance due to output excitation $\left(g_{o 3, \mathrm{fb}}\left[\mathrm{I} / \mathrm{V}^{3}\right]\right)$

(c) Case 2: Third order non-linearity of the RF feedback transconductance due to input excitation $\left(g_{m 3, \mathrm{fb}}\left[\mathrm{I} / \mathrm{V}^{3}\right]\right)$.

(d) Case 2: Third order non-linearity of the RF feedback transconductance due to output excitation $\left(g_{o 3, \mathrm{fb}}\left[\mathrm{I} / \mathrm{V}^{3}\right]\right)$.

In a similar manner, the IM3 output voltage for each of the cases listed above can be written

$$
\begin{gathered}
v_{o 3 a}=-\frac{3}{4} g_{m 3, \mathrm{fb}} Z_{\mathrm{OL} 3} A_{\text {mix }} A_{v 3} H_{\mathrm{hpf} 3} v_{o 1}^{3}-T_{3} v_{o 3 a} \\
v_{o 3 b}=-\frac{3}{4} g_{o 3, \mathrm{fb}} Z_{\mathrm{OL} 3} A_{\text {mix }} A_{v 3} v_{x, \mathrm{ff} 1}^{3}-T_{3} v_{o 3 b} \\
v_{o 3 c}=-\frac{3}{4} g_{m 3, \mathrm{fb}} Z_{\mathrm{OL} 3} A_{\mathrm{mix}} A_{v 3} v_{x, \mathrm{rf} 1}^{3}-T_{3} v_{o 3 c} \\
v_{o 3 d}=-\frac{3}{4} g_{o 3, \mathrm{fb}} Z_{\mathrm{OL} 3} A_{\mathrm{mix}} A_{v 3} v_{e, \mathrm{rf} 1}^{3}-T_{3} v_{o 3 d}
\end{gathered}
$$


Substituting with (16)-(20) in (29)-(32) and collecting terms yields the required IM3 distortion products

$$
\begin{gathered}
v_{o 3 a}=\frac{3}{4} g_{m 3, \mathrm{fb}} Z_{\mathrm{CL} 3} A_{\mathrm{mix}} A_{v 3} H_{\mathrm{hpf} 3}\left(G_{\mathrm{lna}} Z_{\mathrm{CL} 1} A_{\mathrm{mix}} A_{v 1}\right)^{3} A^{3} \\
v_{o 3 b}=-\frac{3}{4} g_{o 3, \mathrm{fb}} Z_{\mathrm{CL} 3} A_{\mathrm{mix}} A_{v 3} H_{\mathrm{hpf} 3}\left(G_{\mathrm{lna}} \frac{Z_{o 1} \| Z_{\mathrm{rf} 1}}{1+T_{1}} A_{\mathrm{mix}} H_{\mathrm{hpf} 1}\right)^{3} A^{3} \\
v_{o 3 c}=\frac{3}{4} g_{m 3, \mathrm{fb}} Z_{\mathrm{CL} 3} A_{v 3} A_{\mathrm{mix}}^{4} H_{\mathrm{hpf} 1}^{3}\left(G_{\mathrm{lna}} Z_{\mathrm{CL} 1} A_{\mathrm{mix}} A_{v 1}\right)^{3} A^{3} \\
v_{o 3 d}=-\frac{3}{4} g_{o 3, \mathrm{fb}} Z_{\mathrm{CL} 3} A_{\mathrm{mix}} A_{v 3}\left(G_{\mathrm{lna}} \frac{Z_{o 1} \| Z_{\mathrm{rf} 1}}{1+T_{1}}\right)^{3} A^{3}
\end{gathered}
$$

\section{ACKNOWLEDGMENT}

This research is supported by the Dutch Technology Foundation (STW). The authors would like to thank STMicroelectronics for Silicon donation and CMP for their assistance. Also thanks go to Gerard Wienk and Henk de Vries of the IC Design Group at the University of Twente. 


\section{REFERENCES}

[1] S. Haykin, "Cognitive radio: brain-empowered wireless communications," IEEE J. Sel. Areas Commun., vol. 23, pp. 201220, Feb. 2005.

[2] D. H. Mahrof, E. A. M. Klumperink, J. Haartsen, and B. Nauta, "On the effect of spectral location of interferers on linearity requirements for wideband cognitive radio receivers," in 4th IEEE Symposium of New Frontiers in Dynamic Spectrum Access Networks (DySPAN), Singapore, Apr. 2010.

[3] B. Razavi, RF Microelectronics, 1st ed. New Jersey: Prentice Hall, 1998.

[4] L. E. Franks and I. W. Sandberg, "An alternative approach to the realization of network transfer functions: The N-path filter," Bell Sys. Tech. J., vol. 39, pp. 1321-1350, Sep. 1960.

[5] K. Koli, J. Jussila, P. Sivonen, S. Kallioinen, and A. Parssinen, “A 900MHz direct $\Delta \Sigma$ receiver in $65 \mathrm{~nm}$ CMOS,” in IEEE Int. Solid-State Circuits Conf. Dig. Tech. Papers, Feb. 2010, pp. 64-65.

[6] A. Mirzaei, H. Darabi, and D. Murphy, "A low-power process-scalable superheterodyne receiver with integrated high-Q filters," in IEEE Int. Solid-State Circuits Conf. Dig. Tech. Papers, Feb. 2011, pp. 60-61.

[7] A. Ghaffari, E. A. M. Klumperink, M. C. M. Soer, and B. Nauta, "Tunable high-Q N-path band-pass filters: Modeling and verification,” IEEE J. Solid-State Circuits, vol. 45, pp. 998-1010, May 2011.

[8] M. Darvishi, R. van der Zee, E. Klumperink, and B. Nauta, "A $0.3 \mathrm{GHz}$ to $1.2 \mathrm{GHz}$ tunable 4 th order switched gm-C band-pass filter with $>55 \mathrm{~dB}$ ultimate rejection and out-of-band IIP3 $=+29 \mathrm{dBm}$," in IEEE Int. Solid-State Circuits Conf. Dig. Tech. Papers, Feb. 2012, pp. 358-359.

[9] S. Youssef, R. van der Zee, and B. Nauta, "Active feedback receiver with integrated tunable RF channel selectivity, distortion cancelling, 48dB stopband rejection and $>+12 \mathrm{dBm}$ wideband IIP3, occupying $<0.06 \mathrm{~mm}^{2}$ in $65 \mathrm{~nm}$ CMOS," in IEEE Int. Solid-State Circuits Conf. Dig. Tech. Papers, Feb. 2012, pp. 166-168.

[10] A. Mirzaei, H. Darabi, J. C. Leete, and Y. Chang, "Analysis and optimization of direct-conversion receivers with $25 \%$ duty-cycle current-driven passive mixers," IEEE J. Solid-State Circuits, vol. 57, pp. 2353-2366, Sep. 2010.

[11] J. Weldon, R. S. Narayanaswami, J. C. Rudell, L. Lin, M. Otsuka, S. Dedieu, L. Tee, K. Tsai, C. Lee, and P. Gray, “A 1.75-GHz highly integrated narrow-band CMOS transmitter with harmonic-rejection mixers," IEEE J. Solid-State Circuits, vol. 36, pp. 2003-2015, Dec. 2001.

[12] Z. Ru, N. A. Moseley, E. A. M. Klumperink, and B. Nauta, "Digitally enhanced software-defined radio receiver robust to out-of-band interference,” IEEE J. Solid-State Circuits, pp. 3359-3375, Dec. 2009.

[13] C. Andrews and A. C. Molnar, "A passive mixer-first receiver with digitally controlled and widely tunable RF interface," IEEE J. Solid-State Circuits, vol. 45, pp. 2696-2708, Dec. 2010.

[14] E. E. Bautista, B. Bastani, and J. Heck, “A high IIP2 downconversion mixer using dynamic matching,” IEEE J. Solid-State Circuits, vol. 35, pp. 1934-1941, Dec. 2000.

[15] M. Hotti, J. Ryynanen, and K. Halonen, "IIP2 calibration methods for current output mixer in direct-conversion receivers," in IEEE Int. Symp. on Circuits and Systems, ISCAS, May 2005, pp. 5059-5062.

[16] B. W. Cook, A. Berny, A. Molnar, S. Lanzisera, and K. S. J. Pister, "Low-power 2.4-GHz transceiver with passive RX front-end and 400-mV supply," IEEE J. Solid-State Circuits, vol. 41, pp. 2757-2766, Dec. 2006.

[17] W. Sansen, Analog Design Essentials, 1st ed. The Netherlands: Springer, 2006.

[18] J. Shi, Y. Z. Xiong, K. Kang, L. Nan, and F. Lin, "RF noise of 65-nm MOSFETs in the weak-to-moderate-inversion region," IEEE Electron Device Lett., vol. 30, pp. 185-188, Feb. 2009.

[19] B. Nauta, "Linear CMOS transconductance element for VHF filters," Electron. Lett., vol. 25, pp. 448-450, Mar. 1989. 
[20] M. Bazes, “Two novel fully complementary self-biased CMOS differential amplifiers,” IEEE J. Solid-State Circuits, vol. 26, pp. 165-168, Feb. 1991.

[21] A. N. Karanicolas, “A 2.7-V 900-MHz CMOS LNA and mixer,” IEEE J. Solid-State Circuits, vol. 31, pp. 1939-1944, Dec. 1996.

[22] D. K. Shaeffer and T. H. Lee, “A 1.5-V 1.5-GHz CMOS low noise amplifier," IEEE J. Solid-State Circuits, vol. 32, pp. 745-759, May 1997.

[23] F. Gatta, E. Sacchi, F. Svelto, P. Vilmercati, and R. Castello, "A 2-dB noise figure 900-MHz differential CMOS LNA," IEEE J. Solid-State Circuits, vol. 36, pp. 1444-1452, Oct. 2001.

[24] B. G. Perumana, J. C. Zhan, S. S. Taylor, B. R. Carlton, and J. Laskar, "Resistive feedback CMOS low-noise amplifiers for multiband applications," IEEE Trans. Microw. Theory Tech., vol. 56, pp. 1218-1225, May 2008.

[25] J. C. Zhan and S. S. Taylor, "A 5GHz resistive-feedback CMOS LNA for low-cost multi-standard applications," in IEEE Int. Solid-State Circuits Conf. Dig. Tech. Papers, Feb. 2006, pp. 721-730.

[26] F. Bruccoleri, E. A. M. Klumperink, and B. Nauta, "Wide-band CMOS low-noise amplifier exploiting thermal noise canceling," IEEE J. Solid-State Circuits, vol. 39, pp. 275-282, Feb. 2004.

[27] S. C. Blaakmeer, E. A. M. Klumperink, D. M. W. Leenaerts, and B. Nauta, "Wideband balun-LNA with simultaneous output balancing, noise-canceling and distortion-canceling," IEEE J. Solid-State Circuits, vol. 43, pp. 1341-1350, Jun. 2008.

[28] G. Palumbo and S. Pennis, Feedback Amplifiers: Theory and Design, 1st ed. New York: Kluwer Academic Publishers, 2003.

[29] A. J. Annema, B. Nauta, R. Langevelde, and H. Tuinhout, "Analog circuits in ultra-deep-submicron CMOS," IEEE J. Sel. Areas Commun., vol. 40, pp. 132-143, Jan. 2005.

[30] A. Safarian, A. Shameli, A. Rofougaran, M. Rofougaran, and F. D. Flaviis, "Integrated blocker filtering RF front ends," in Proc. IEEE Radio Frequency Integr. Circuits Symp., Jun. 2007, pp. 13-16.

[31] T. Werth, C. Schmits, R. Wunderlich, and S. Heinen, "An active feedback interference cancellation technique for blocker filtering in RF receiver front-ends," IEEE J. Solid-State Circuits, vol. 45, pp. 989-997, May 2010.

[32] H. Darabi, "A blocker filtering technique for SAW-less wireless receivers," IEEE J. Solid-State Circuits, vol. 42, pp. 2766-2773, Dec. 2007. 

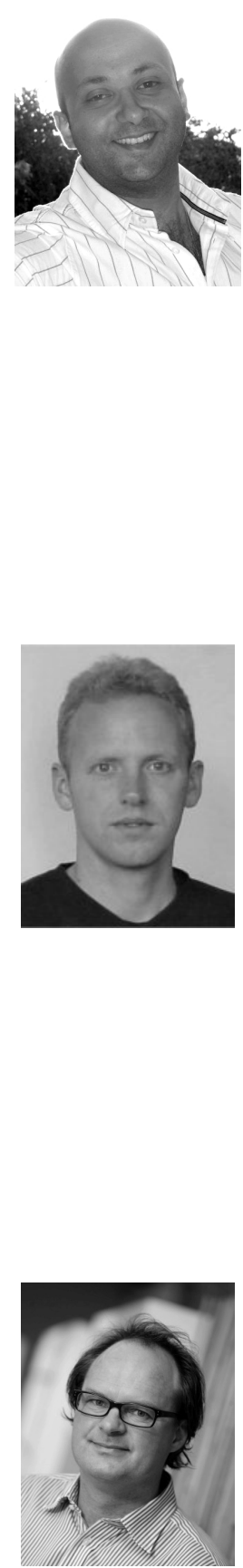

Bram Nauta was born in Hengelo, The Netherlands. In 1987 he received the M.Sc degree (cum laude) in electrical engineering from the University of Twente, Enschede, The Netherlands. In 1991 he received the $\mathrm{Ph}$.D. degree from the same university on the subject of analog CMOS filters for very high frequencies. In 1991 he joined the Mixed-Signal Circuits and Systems Department of Philips Research, Eindhoven the Netherlands. In 1998 he returned to the University of Twente, as full professor heading the IC Design group. His current research interest is high-speed analog CMOS circuits.

He served as Associate Editor of IEEE Transactions on Circuits and Systems II (1997-1999). He was Associate Editor(20012006) and later the Editor-in-Chief (2007-2010) of IEEE Journal of Solid-State Circuits. He is member of the technical program committees of the International Solid State Circuits Conference (ISSCC) where he is the 2013 Program Committee Chair, the European Solid State Circuit Conference (ESSCIRC), and the Symposium on VLSI circuits. He is co-recipient of the ISSCC 2002 and 2009 "Van Vessem Outstanding Paper Award", is distinguished lecturer of the IEEE, elected member of IEEE-SSCS AdCom and is IEEE fellow. 